\title{
Asymmetrical choice-related ensemble activity in direct and indirect- pathway striatal neurons drives perceptual decisions
}

Lele Cui ${ }^{1,2,6}$, Shunhang Tang ${ }^{1,2,6}$, Kai Zhao ${ }^{4}$, Jingwei Pan ${ }^{1}$, Zhaoran Zhang, Bailu $\mathrm{Si}^{4}$, Ninglong $\mathrm{Xu}^{1,2,3 *}$

${ }^{1}$ Institute of Neuroscience, State Key Laboratory of Neuroscience, CAS Center for Excellence in Brain Science and Intelligence Technology, Chinese Academy of Sciences, Shanghai 200031, China

${ }^{2}$ School of Future Technology, University of Chinese Academy of Sciences, Beijing 100049, China

${ }^{3}$ Shanghai Center for Brain Science and Brain-Inspired Intelligence Technology, Shanghai 201210, China

${ }^{4}$ School of Systems Science, Beijing Normal University, Beijing, 100875, China

${ }^{5}$ School of Life Sciences, Sichuan University, Chengdu, Sichuan 610064, China

${ }^{6}$ These authors contributed equally

*Correspondence: xunl@,ion.ac.cn 
Action selection during decision-making depends on the basal ganglia circuits that comprise the direct and indirect pathways known to oppositely control movement. However, the mechanism for coordinating these opponent pathways during decisionmaking remains unclear. We address this by employing deep-brain two-photon imaging and optogenetic manipulations of the direct- and indirect-pathway spiny projection neurons (dSPNs and iSPNs) in the posterior striatum during an auditory decisionmaking behavior. We show that while dSPNs and iSPNs play opposite causal roles during decision-making, each subtype contains divergent ensembles preferring different choices. The ensembles in dSPNs show stronger contralateral dominance than those in iSPNs manifested by higher-level activation and synchronization. Consistent with this asymmetrical contralateral dominance, optogenetic disinhibition of both pathways promoted contralateral choices. A computational model incorporating the striatal ensemble asymmetry recapitulated the causal behavioral effects. Our results uncover the asymmetry between opponent SPN ensembles as a circuit mechanism for action selection during decision-making.

In a dynamic environment organisms need to make reliable and accurate movements based on diverse sensory inputs, a process known as sensory-guided action selection or perceptual decision-making. In the mammalian brain, perceptual decision-making critically depends on an evolutionarily conserved brain structure, the basal ganglia, a group of subcortical nuclei that are widely implicated in movement control and decision-making ${ }^{1-8}$. The striatum is the main input structure of the basal ganglia, receiving excitatory input from virtually the entire cortical and thalamic regions ${ }^{9-11}$ as well as strong dopaminergic input from the midbrain, and providing output to downstream basal ganglia nuclei to influence behavioral output, and is therefore well poised as a key node in the decisionmaking networks.

The two major subtypes of projection neurons in the striatum, the dSPNs and iSPNs, give rise to the direct and indirect basal ganglia pathways by innervating distinct downstream nuclei. In the classical models ${ }^{1,2,4,8}$, the two striatal pathways play opposing 
roles in movement control, with the direct pathway promoting movements, while the indirect pathway suppressing movements. These models suggest that the direct pathway tends to be active and the indirect pathway inactive during actions ${ }^{3,6,8}$. However, recent optical and electrical recordings from genetically defined subtypes of SPNs showed that both the direct and indirect pathways were concurrently active during movements, often showing indistinguishable activity patterns ${ }^{12-16}$. To date, it has been a lack of consensus on how the opposing basal ganglia pathways work together to produce coherent movements ${ }^{3,6}$. In the context of decision-making, how the two opponent pathways coordinate in transforming sensory input to consistent motor output is thus an outstanding question yet to be addressed.

One possibility is that in different types of behaviors, the dSPNs and iSPNs, while being concurrently active, do not necessarily show identical activity patterns. For instance, they could differentiate in either activation time or in response amplitude ${ }^{3,6}$. It is therefore important to directly examine the ensemble activity patterns of dSPNs and iSPNs during decision-making behavior to probe the coordination mechanism, and to test how such mechanism may influence decision-making behavior. Here we employed in vivo deep-brain two-photon imaging to directly record single-cell level activity over a large population of dSPNs and iSPNs during a precisely controlled auditory-guided decision-making task ${ }^{17,18}$. We focus on the posterior tail of the striatum (TS) which has been shown to receive direct input from auditory cortex ${ }^{10,19,20}$. We found that both dSPNs and iSPNs comprise subpopulations with opposing preferences to either contralateral or ipsilateral choices. These subpopulations in dSPNs and iSPNs exhibit ensemble-level contralateral dominance. Importantly, such contralateral dominance is stronger in dSPNs than iSPNs in the time epoch preceding choice execution. This represents a task-related asymmetry in the ensemble-level activity between dSPNs and iSPNs that may support contralateral action selection. Using cell-type specific bidirectional optogenetic manipulations, we found that dSPNs and iSPNs play opposite causal roles in the auditory decision-making behavior. Interestingly, concurrent disinhibition of both dSPNs and iSPNs via inhibiting striatal pavalbumin (PV) interneurons biased choice behavior toward the contralateral side, a causal effect agreeing with the asymmetrical ensemble activity. Finally, we constructed a 
computational model incorporating the observed asymmetrical activity patterns of dSPNs and iSPNs. This model recapitulated the causal behavioral effects of dSPNs and iSPNs found in our optogenetic experiments. Thus, these findings support that the task-dependent asymmetrical activity patterns in dSPN and iSPN ensembles serve as a circuit-level coordination mechanism in the basal ganglia controlling choice behavior during decisionmaking.

\section{Results}

\section{Imaging single-neuron activity of dSPNs and iSPNs in posterior striatum during decision-making task}

We trained head-fixed mice to perform an auditory-guided decision-making task ${ }^{17,18}$. Mice were required to classify sound stimuli (pure tones between 5 and $20 \mathrm{kHz}$ ) as belonging to high or low frequency categories (Fig. 1a; Movie S1). The categorical choices were quantified as the probability of licking the left or right lick port in response to various tone frequencies (Fig. 1b), which allowed us to obtain psychophysics level measurement of perceptual decision-making behavior (Fig. 1c). To examine the relationship between striatal neuron activity and the auditory decision behavior we focused on the posterior tail of the striatum (TS) that has been shown to receive input primarily from sensory cortices ${ }^{9,10}$ and was implicated in auditory-related choice behavior ${ }^{20,21}$. We performed in vivo two-photon calcium imaging from individual dSPNs or iSPNs respectively through a cannular imaging window chronically implanted in TS (Extended Data Fig. 1; Methods). Genetically encoded calcium sensor GCaMP6s was expressed selectively in dSPNs or iSPNs by injecting AAVhSyn-FLEX-GCaMP6s in TS of either D1-cre or D2-cre transgenic mice (Fig. 1d; Methods). Task related calcium signals with high signal to noise ratio from individual neurons were reliably detected (Fig. 1e, f). We found that activity in individual neurons exhibited clear task modulation, often showing markedly different responses to different choices regardless of stimulus category. Neurons showing preferred responses to either left or right choices (on the contra- or ipsilateral side of the imaged hemisphere) often coexist in simultaneously imaged dSPNs or iSPNs (Fig. 1g-j), indicating within-subtype heterogeneity. 


\section{Asymmetrical contralateral preference in dSPNs and iSPNs preceding action execution}

The classical rate model postulates that the direct pathway should be more strongly active than the indirect pathway when eliciting desired movements ${ }^{1,3,8}$. However, recent studies reported that both the dSPNs and iSPNs were concurrently active and often show indistinguishable activity patterns during spontaneous movements ${ }^{12,13,16,22,23}$. One possibility is that the coordination of the direct and indirect pathways can be more complex than that expected from earlier models, and there could be nuanced specificity in the activity patterns of dSPNs and iSPNs that may depends on specific behavioral contexts ${ }^{3,6,14,15,24}$. Here we examine whether the dSPNs and iSPNs may show differential activity patterns at the population level during perceptual decision-making behavior.

We first examined the single-neuron encoding of choice and sensory information across all imaged neurons for both dSPNs and iSPNs. We used Receiver Operating Characteristic (ROC) analysis to examine the selectivity in the calcium signals preceding mice's choice (answer lick time), and defined a selectivity index (SI) to measure the neuronal preference for choices and sensory categories. A positive SI value was defined as preferring the contralateral side, and a negative SI value was defined as preferring ipsilateral side. Significant SI values with the same sign in both correct and error trials would indicate selectivity for choices, whereas significant SI values with opposite signs in correct and error trials would indicate selectivity for sensory categories (Fig. 2a, c; Methods). We found that the majority of neurons in both dSPNs and iSPNs are selective to choices (Fig. 2a, c). Interestingly, both dSPNs and iSPNs contains divergent subpopulations showing significant selectivity to either contralateral or ipsilateral choices (Fig. 2a, c; Extended Data Fig. 2). This indicates that there is within-cell type heterogenous ensembles coding for competing choices. Such heterogeneous ensemble organization raises the possibility that previously observed concurrent activation of dSPNs and iSPNs can be due to the activation of different subpopulations of dSPNs and iSPNs preferring the same motor actions, but not necessarily due to uniform co-activation of dSPNs and iSPNs. This distinction is important since it 
suggests that the coordination of the direct and indirect pathways can involve more complex competition and cooperation among multiple striatal ensembles from the two hemispheres.

To examine the potential relative contributions to decision execution from different subpopulations, we analyzed the response amplitude in all SPNs showing significant choice selectivity prior to animals' answer time. Overall, there are higher proportions of contralateral preferring neurons (dSPNs, 275/811, 34\%; iSPNs, 307/1152, 27\%) than ipsilateral preferring neurons (dSPNs, 155/811, 19\%; iSPNs, 125/1152, 11\%) (Fig. 2b, d). By averaging the standard calcium signals across neurons with the same choice preference, we found that the amplitude of responses to preferred choices are stronger in contralateral preferring neurons than in ipsilateral preferring neurons, both in dSPNs (Fig. 2b, upper left vs. lower right) and in iSPNs (Fig. 2d, upper left vs. lower right). Therefore, the contralateral preferring subpopulations are more dominant both in dSPNs and iSPNs, which would imply that the major contribution to motor choices would arise from the competition between contralateral preferring dSPNs and iSPNs. We thus examined the relative strength between these two subpopulations. As shown in Fig. 2e, the activation level in dSPNs during contralateral trials is significantly greater than that in iSPNs (see also Fig. 2q). Consistently, the contralateral preference (difference between contra- and ipsilateral responses) is also significantly stronger in dSPNs than in iSPNs (Fig. 2f). In contrast, the difference between ipsilateral preferring dSPNs and iSPNs is relatively minor in their ipsilateral responses (Fig. 2g, q) and in their ipsilateral preferences (difference between ipsi- and contralateral responses; Fig. 2h). Taken together, our single-cell resolution population imaging reveals that while multiple subpopulations of SPNs form a complex competition and cooperation system, the dSPNs and iSPNs exhibit ensemble-level asymmetry: higher contralateral dominance in dSPNs than in iSPNs. Such asymmetry arose before the decision execution time, and can thus potentially support the contralateral choices.

To illustrate the temporal property of the response asymmetry between dSPNs and iSPNs, we performed deconvolution of the calcium signals to infer the underlying spike rate changes (Methods). We found that the peaks of spike rate changes as well as the spike ratebased choice preferences are indeed preceding the answer time, with contralateral preference greater in dSPNs than in iSPNs (Fig. 2i-p, and r). Thus, the response asymmetry, i.e., 
stronger contralateral dominance in dSPNs than in iSPNs, already reached peak level before decision execution, and thus is likely to contribute to contralateral action selection.

Our single-cell resolution population imaging thus revealed a multi-ensemble organization of the striatal projection neurons with asymmetry between dSPNs and iSPNs: the dSPNs showing higher contralateral dominance than iSPNs. This represents an ensemblelevel mechanism coordianting the two opponent pathways for action selection during decision-making.

\section{The dSPNs and iSPNs exhibit asymmetrical spatiotemporal activity patterns}

Response strength not only manifests in the firing rate of individual neurons, but also in population-level synchronization between neurons. Our single-cell resolution two-photon imaging allows for examining the spatiotemporal activity patterns in simultaneously imaged neurons. We thus examined whether there was asymmetry between dSPNs and iSPNs in their spatiotemporal activity patterns during the task. We found that for both dSPNs and iSPNs, nearby neurons showed greater temporal correlation than more distant neurons, with a monotonic dependence on inter-neuronal spatial distances (Fig. 3a-g). This is consistent with a previous study using endoscopic imaging during self-paced movements ${ }^{22}$. Here we further compared the spatiotemporal activity patterns between dSPNs and iSPNs. We used two-way repeated-measures ANOVA to simultaneously examine the dependence of inter-neuronal correlation on spatial distance and on cell types. We found that in addition to the dependence on inter-neuronal distance, the temporal correlations were significantly stronger in dSPNs than in iSPNs (Fig. 3g). For the functional subpopulations preferring the same choices, inter-neuronal temporal correlations were also significantly stronger in dSPNs than in iSPNs (Fig. 3h, i). For the different functional subpopulations within each cell type, the temporal correlations were significantly higher in contralateral preferring neurons than in ipsilateral preferring neurons for both dSPNs (Fig. 3j) and iSPNs (Fig. 3k). Thus, consistent with the stronger contralateral dominance in dSPNs than iSPNs shown in response amplitude, dSPNs also show higher activity synchrony than iSPNs, which is likely to have greater impact on the 
downstream neurons.

\section{The contralateral dominance in dSPNs is preserved in the axonal activity in the downstream region}

The stronger contralateral response amplitude as well as higher temporal synchrony in dSPN ensembles would produce stronger output at the downstream brain regions. We thus examined the population activity of dSPN axons in the downstream substantia nigra pars reticulata (SNr). We expressed GCaMP6s in dSPNs in TS and recorded the axonal calcium signals in SNr using fiber photometry during task performance (Fig. 4a; Methods). The spatial separation between $\mathrm{SNr}$ and TS allows us to optically measure the activity of dSPNs axons in $\mathrm{SNr}$ without the influence from somatic signals. We observed reliable axonal calcium signals showing clear preferences for choices (Fig. 4b). Across recording sites, the dSPN axons in SNr showed significantly stronger responses in contralateral trials than in ipsilateral trials (Fig. 4c). Similar to the ROC-based selectivity index in somatic responses as shown in Fig. 2a, in all the recording sites, the axonal activity showed significant selectivity to choice directions consistent in correct and error trials (Fig. 4d), indicating selective coding for choice but not for sensory information. Across recording sites, the ROC-based selectivity indices were also stronger for the contralateral choices $(9 / 12, \mathrm{p}<0.05)$. Thus, consistent with the dominant contralateral choice preference and temporal synchrony in the soma of contralateral preferring dSPNs, the axonal activity in the output region of basal ganglia also show greater contralateral choice preference.

\section{Optogenetic activation of the direct and indirect pathways oppositely regulate perceptual decisions}

To examine how the information encoded in different subtypes of SPNs impacts perceptual decisions, we examined the causal roles of the striatal SPNs during task performance. First, we performed unilateral optogenetic activation of either dSPNs or iSPNs during the task. To activate dSPNs, we expressed Channelrhodopsin-2 (ChR2) in dSPNs in a Cre-dependent manner by injecting AAV-CAG-FLEX-ChR2 in the TS of D1-Cre mice followed by implanting fiber optics above the injection sites. During experiments, photostimulation (473 
$\mathrm{nm}$ laser) was delivered to unilateral TS to activate dSPNs on a subset $(\sim 30 \%)$ of trials randomly interleaved with control trials without photostimulation (Fig. 5a and Extended

Data Movie 2; Methods). We found that activation of dSPNs in the TS strongly biased animals' choices toward the contralateral side relative to the photostimulated hemisphere (Fig. 5b). The trial-by-trial design with photostimulation in a minor fraction of trials allows us to examine the effect of dSPN activity on the current-trial decision without confounding from general reinforcement changes. Indeed, the increase of contralateral choices was not accompanied by any significant change in miss rate, suggesting that there was no significant change in motivation level (Extended Data Fig. 4b). Moreover, the increase of contralateral choices was not due to direct motor drive of licking, since the lick rate in individual trials was not significantly different between photostimulation and control trials (Extended Data Fig. 5b). Thus, dSPN activation promoted the contralateral choice during perceptual decisionmaking.

To activate iSPNs we injected AAV-CAG-FLEX-ChR2 in the TS of D2-Cre mice (Fig. 5c; Methods). We found that unilateral activation of iSPNs strongly biased animals' choices toward ipsilateral side relative to the photostimulated hemisphere (Fig. 5d), an effect opposite to that of dSPN activation. Similar to dSPN activation, we found no significant changes in miss rate during photostimulation (Extended Data Fig. 4c), ruling out potential influence of motivational state following iSPN activation. This result also suggests that rather than general suppression of movement, iSPNs can actively participate in the decision process of selecting from competing actions.

An advantage of optogenetics is the precise control of photostimulation time, which would allow for examining the temporal specificity of the effect of neuronal activation on behavioral performance. We demonstrate this by applying temporally confined photostimulation to the activation of iSPNs in D2-Cre mice. We wondered whether iSPNs activation mainly affected the sensorimotor decision process per se or the post-decision motor execution. Although the time of decision commitment cannot be explicitly determined, we observed that mice often make correct answer lick shortly after the onset and before the end of sound stimulus (e.g., see Fig. 1b). For the D2-Cre mice shown in Fig. 5d, the first quartile of the answer lick time is $\sim 150$ ms (Extended Data Fig. 6a), suggesting 
that the earliest possible decision commitment could be well before the end of sound stimulus. We thus confined the photoactivation of iSPNs within a $150 \mathrm{~ms}$ window after sound stimulus onset, which would cover the potential sensory decision period while avoiding the majority of answer lick (Fig. 5e). We found that this manipulation also strongly biased animals' choice toward the ipsilateral side. Conversely, when we confined photoactivation within the second half $(150 \mathrm{~ms})$ of the sound stimulus period where mice often make putative post-decision answer licks, the choice behavior was largely unaffected (Fig. 5g). Together, these results suggest that photoactivation of iSPNs mainly influences the sensorimotor decision process but not the post-decision motor execution. To rule out potential non-specific pre-decision effect, we delivered the photostimulation in an intertrial-interval epoch before the sound stimulus onset, and found no significant changes in the behavioral performance (Fig. 5h).

\section{Optogenetic inactivation reveals opposing contributions to choice behavior by dSPNs and iSPNs}

To examine the respective contributions of dSPNs and iSPNs to perceptual decisions, we further used optogenetic inactivation to separately examine the involvement of dSPNs and iSPNs in the task performance. To inactivate dSPNs, we injected AAV-Ef1 $\alpha$-DIO-eNpHR3.0 in the TS of D1-Cre mice to express eNpHR3.0 in dSPNs, and delivered unilateral photostimulation $(593 \mathrm{~nm}$ ) through fiber optics on interleaved trials during each behavioral session (Fig. 5i). We found that inactivation of dSPNs significantly biased animals' choice toward the ipsilateral side relative to the photostimulated hemisphere (Fig. 5j). This is consistent with our observations of ensemble-level contralateral dominance of dSPN activity (Fig. 2) and the contralateral choice promotion by dSPN activation (Fig. 5b). To inactivate iSPNs, we injected AAV-Ef1 $\alpha$-DIO-eNpHR3.0 in the TS of D2-cre mice to express eNpHR3.0 in iSPNs (Fig. 5k). We found that iSPN inactivation led to a slight but significant bias of animals' choice toward the contralateral side (Fig. 5l, $P=1.7 \times 10^{-4}$, two-way repeated-measures ANOVA). This result is also consistent with the expectation following the iSPN imaging (Fig. 2) and activation results (Fig. 5d). To confirm the relatively weak 
behavioral effect following iSPN inactivation, we expressed the soma-targeted Guillardia theta anion-conducting channelrhodopsin-2 (stGtACR2), a more potent activity suppressor ${ }^{25}$, in iSPNs, and delivered blue laser $(473 \mathrm{~nm})$ to inactivate iSPNs during task performance (Fig. 5m). We found similar effect of contralateral bias following iSPN inactivation by stGtACR2 (Fig. 5n) as that following inactivation using eNpHR3.0. Similar to optogenetic activation, unilateral inactivation of dSPNs or iSPNs did not influence the miss rate or lick rate (Extended Data Fig. 4d-i), ruling out potential effects on motivation or tongue movement. Thus, unilateral inactivation of dSPNs or iSPNs revealed opposing contributions to choice behavior, consistent with the expectations based on our imaging and activation results.

\section{Concurrent disinhibition of dSPNs and iSPNs promotes contralateral choice}

Our two-photon imaging results show that despite concurrent activation of different subpopulations of dSPNs and iSPNs, the dSPNs exhibited overall stronger responses than iSPNs for the contralateral choice, suggesting that concurrent activation of the ensembles in the two subtypes of SPNs may still support the choice of contralateral action. We explored this possibility by manipulating a common inhibitory input from local parvalbumin (PV) expressing interneurons ${ }^{26-29}$. Previous studies showed the inhibitory input from PV interneurons to dSPNs and iSPNs was comparable ${ }^{29}$, and inactivation of striatal PV interneurons increased SPN activity level ${ }^{28}$. We thus reasoned that experimentally inhibiting PV interneurons would lead to concurrent disinhibition of both dSPNs and iSPNs, mimicking coactivation with physiological excitatory inputs preserved. This would allow us to test the behavioral effect of relatively physiological coactivation of the two pathways. We first examined whether striatal PV interneurons show any task-related activity by recording their calcium signals using fiber photometry in a cell-type specific manner during task performance (Fig. 6a). We expressed GCaMP6s in PV interneurons by injecting AAV-hSynFLEX-GCaMP6s in the TS of PV-Cre mice, and implanted fiber optics above the injection sites. We found that PV interneurons in TS are strongly activated during the choice period (Fig. 6b), indicating that PV interneurons are actively involved in the regulation of striatal 
circuits during choice behavior. Furthermore, we found that PV interneurons showed comparable responses to contra- and ipsilateral choices (Fig. 6c), suggesting that their overall excitatory inputs coding for opposing choices are balanced. This result implies that experimentally inhibiting PV interneurons would lead to a disinhibitory effect on both subtypes of SPNs comparable for ipsi- and contralateral choices.

We thus performed optogenetic inactivation of the striatal PV interneurons by expressing eNpHR3.0 in these neurons using AAV-Ef1a-DIO-eNpHR3.0 in PV-Cre mice (Fig. 6d). We found that unilateral inactivation of PV interneurons in the TS significantly biased animals' choices toward the contralateral side (Fig. 6e, $P=3.1 \times 10^{-3}$, two-way repeated-measures ANOVA). Taken together, these results indicate that concurrent disinhibition of dSPNs and iSPNs promotes the contralateral choice, and thus support the convergent prediction from both our imaging and optogenetic results that concurrent activation of dSPNs and iSPNs with physiological excitatory inputs support contralateral action selection following decision-making.

\section{Computational implementation of the coordination among SPN ensembles}

Based on our imaging data (Fig. 2), both dSPNs and iSPNs comprise divergent functional subpopulations with response preferences to competing choices. The competition and cooperation among these functional subpopulations from both hemispheres would determine the final behavioral choices. To explore the computational plausibility of such coordination mechanism, we constructed a network model of the basal ganglia circuitry incorporating the multiple functional subpopulations of SPNs in the two hemispheres (Fig. 7a). In this model, the sensory decisions are derived from cortex and sent to dSPNs, iSPNs and striatal PV neurons via cortico-striatal projections ${ }^{30}$. Within the local striatal region, PV neurons provide inhibitory input to both dSPNs and iSPNs, and the two subtypes of SPNs provide mutual inhibitory input to each other ${ }^{27,29}$. The dSPN and iSPN populations provide either inhibitory or excitatory (indirectly) input to ipsilateral SNr through the direct and indirect pathways respectively ${ }^{2,31}$. The left and right $\mathrm{SNr}$ populations compete with each other, and the dominant $\mathrm{SNr}$ population determines the left or right choices. The 
weights of cortical input to different subpopulations of SPNs were set according to their relative response strengths observed in our imaging experiments (Extended Data Tab. 1). These settings allowed the model to recapitulate the relative response strengths in the simulated SPN activity as in our experimental data (Fig. 7b, c).

To examine the computational plausibility for the coordination of multiple SPN subpopulations to produce expected choice behavior, we asked whether our network model could recapitulate the perceptual decision behavior under both control condition and celltype specific manipulation conditions. First, simulation using this model was able to recapitulate the choice behavior with similar psychometric functions as in behavioral data (Fig. 7d-h). Importantly, when we removed the asymmetry between dSPNs and iSPNs in silico, the model was no longer able to produce the choice behavior based on sensory stimuli (Fig. 7I). Next, we used this model to simulate optogenetic manipulations by increasing or decreasing the activity level of different striatal cell types (Extended Data Tab. 2). We found that unilateral increasing of dSPN activity biased the choice towards the contralateral side, whereas unilateral increasing of iSPN activity biased the choice towards the ipsilateral side (Fig. 7d, e), recapitulating our experimental results of optogenetic activation of dSPNs and iSPNs (Fig. 5a-d). Unilateral decreasing of dSPN activity significantly biased the choice towards ipsilateral side, whereas unilateral decreasing of iSPN activity significantly biased the choice towards contralateral side (Fig. 7f, g), which also recapitulated our optogenetic inactivation results (Fig. 5i-n). Finally, we simulated the concurrent disinhibition by decreasing the activity of unilateral PV interneurons, which provide inhibitory input to both dSPNs and iSPNs with similar connectivity weights in the model (Extended Data Tab. 1 and Tab. 2). Decreasing unilateral PV interneuron activity biased the choice towards the contralateral side (Fig. $7 \mathbf{h}$ ), which recapitulated our experimental observation following PV neuron inactivation (Fig. 6e). These simulation results demonstrate the computational plausibility that the coordination among different SPN subpopulations with ensemble-level asymmetry between dSPNs and iSPNs supports the sensory-guided choice behavior. 


\section{Discussion}

By combining cell-type specific in vivo two-photon imaging and optogenetic manipulations with a psychophysical decision-making task, we show that the posterior tail of the striatum contains functionally divergent subpopulations of SPNs, and the coordination of these subpopulations plays an essential role in sensory-guided decision-making. Our single-cell resolution imaging reveals that both dSPNs and iSPNs contain subpopulations with divergent preferences to competing choices, consequentially resulting in concurrent activation of different ensembles of both dSPN and iSPN in response to the same motor choices (Fig. 2). This is consistent with previously observed coactivation of the direct and indirect pathways during movement. Importantly, our imaging data also reveal that the dSPNs and iSPNs show ensemble-level asymmetry in their contralateral choice preference during decision-making, which can be essential in driving the decision execution.

Corroborating the asymmetry in contralateral dominance in the response amplitude, dSPNs show higher level of population synchronization than iSPNs (Fig. 3), which can lead to stronger impact to downstream neurons. Cell-type specific optogenetic manipulations of dSPNs and iSPNs reveal opposing effects on choice behavior with the temporal specificity in the decision-relevant period (Fig. 5). These manipulation results extend the classic notion of the differential roles of the direct and indirect striatal pathways in movement control to the domain of perceptual decision-making with trial-by-trial specificity and subtrial precision. Moreover, concurrent optogenetic disinhibition of dSPNs and iSPNs led to significant contralateral bias of choice behavior, as predicted by the asymmetrical ensemble preferences of dSPNs and iSPNs during task performance. This suggests that physiological level coactivation of dSPNs and iSPNs can drive desired motor choices, given the asymmetry between the two pathways. Finally, computational simulations using a neural circuit model featuring the multi-subpopulation of SPNs with the asymmetry between dSPN and iSPN activity recapitulated the perceptual decision-making behavior as well as the behavioral effects of cell-type specific optogenetic manipulations (Fig. 7), suggesting that it is computationally plausible for our observed population activity patterns to support decision-making behavior. 
We focus on the posterior tail of the striatum (TS) because this region receives auditory sensory input ${ }^{9,10,20,21,32,33}$. The TS is thus more likely to be directly involved in sensorimotor decision process comparing to the more anterior dorsomedial striatum (DMS), which is more likely to be involved in movement control and reward-based learning ${ }^{12,34,35}$. To confirm the region specificity, we performed optogenetic silencing in both the TS and the DMS, and found that unilateral inactivation of TS but not DMS significantly impaired the auditory-guided contralateral choice (Extended Data Fig. 7).

Although SPNs in the TS receive synaptic input from auditory regions, we found that only a small number of neurons showed sensory selectivity, while the majority of neurons of both dSPNs and iSPNs showed strong selectivity for choices (Fig. 2a, c). This can be partially due to that the calcium signals detected using GCaMP6 primarily reflect strong spiking activity related to decision readout. We verified this coding preference for choice information by recording the axonal activity of dSPNs using fiber photometry in the downstream $\mathrm{SNr}$ and also found primarily choice selective responses with the contralateral dominance (Fig. 4), consistent with the imaging results from the somatic region in TS. This is also consistent with a previous study using fiber photometry to record GCaMP6 signals from dSPNs during a visual detection task showing predominantly licking-related signals instead of visual responses ${ }^{36}$.

Recent studies reported coactivation of both the dSPNs and iSPNs during movement $12,13,16,22,23$. These observations appear to contradict the direct prediction from earlier models derived from the opposing effects on movement of the direct and indirect pathways $^{1,3,8}$. Such disagreement could be potentially reconciled if there were certain level of differentiation in the activity patterns between dSPNs and iSPNs ${ }^{3,6,24}$. Here we found that dSPNs and iSPNs exhibit ensemble-level differences in the contralateral dominance during choice behavior, with stronger contralateral population responses in dSPNs preceding decision execution (Fig. 2). These observations are also consistent with recent studies reporting a difference in the bulk activity between dSPNs and iSPNs recorded using fiber photometry during a turning behavior ${ }^{14}$, and the differential subthreshold activity of dSPNs and iSPNs during a tactile detection task ${ }^{15}$. The differentiation in the dSPN and iSPN activity implies that while concurrently active, the stronger contralateral dominance of dSPN 
ensemble activity would still support a selected contralateral action. In agreement with this possibility, a previous study showed that concurrent over-activation of both direct and indirect striatal pathways biased the animals' locomotion towards the contraversive direction ${ }^{16}$. Here, instead of over-activation, we concurrently disinhibited both striatal pathways by optogenetic inactivation of PV interneurons, a common source of inhibition to dSPNs and iSPNs, which would elevate the activity of both types of SPNs in a more physiological manner. This manipulation significantly biased animals' choice towards the contralateral side, consistent with the prediction from the asymmetrical ensemble activity found in our imaging data. Furthermore, using a neural circuit model, we demonstrate that the asymmetry in dSPN and iSPN activity is computationally important to produce the sensoryguided choice behavior (Fig. 7). Thus, the concurrent yet asymmetrical activity in dSPNs and iSPNs represents a coordination mechanism for the opponent basal ganglia pathways to control action selection during decision-making.

The causal roles of the cell-type specific striatal circuits have been widely studied in various forms of motor function, including locomotion ${ }^{23,37}$, sequential actions ${ }^{38,39}$, and movement kinematics ${ }^{40}$. For sensory-guided decision-making, however, the causal roles of specific striatal pathways are less well-understood. A recent study on a visual detection go/no go task showed that activation of dSPNs and iSPNs both led to increased licking upon visual stimulation, with a stronger effect when the visual stimulus was on the contralateral side for dSPN activation ${ }^{36}$. But it was intriguing that activating iSPNs and dSPNs both increased licking behavior given their opponent roles in movement control ${ }^{3}$. This might be attributable to the single-action task design. Here, our two-choice task allows for disambiguating the distinct lateralized causal roles for both dSPNs and iSPNs. We found that activation of dSPNs and iSPNs elicited strong but oppositely lateralized decision-bias (Fig. 5), consistent with the general notion of the functional opponency of the direct and indirect striatal pathways. In addition, since activation normally does not reproduce physiological activity patterns, it is important to also examine the effect of inactivation in order to reveal the causal involvement of neuronal activity. Here, we found that optogenetic inactivation of dSPNs and iSPNs led to opposite lateralized biasing of choice behavior with reversed directions than the activation effects (Fig. 5), revealing the 
causal involvement of the two striatal pathways in decision-making behavior.

Two-photon calcium imaging allows for examining inter-neuronal correlations in simultaneously imaged large populations with both cell-type specificity and single-cell level spatial information. Our analysis of population neuronal synchrony was based on a previous study imaging SPNs using one-photon microendoscope in freely moving mice ${ }^{22}$. Consistent with the previous observation of the spatiotemporal patterns of SPNs, we also found a monotonic dependence of the inter-neuronal correlation on the spatial distance between neurons. More importantly, while in the self-paced freely moving mice, it can be challenging to compare the population synchrony between dSPNs and iSPNs given idiosyncratic movement patterns in individual animals ${ }^{22}$, here in the well-trained sensorimotor decision task in head-fixed mice, the population synchrony in dSPNs or iSPNs was relatively consistent across different behavioral sessions, which allowed us to discern a significant trend with higher inter-neuronal correlations in dSPNs than in iSPNs

(Fig. 3). Such difference is consistent with stronger population firing rates in dSPNs corroborating the asymmetry in ensemble response preferences between dSPNs and iSPNs.

The asymmetry in the ensemble response patterns between dSPNs and iSPNs could be attributed to the differential synaptic strength in the cortical or thalamic input to different subtypes of SPNs, or could also be due to lateralized dopaminergic input ${ }^{41}$, and may depend on dopamine-dependent plasticity ${ }^{42}$. The mechanism for the regulation of synaptic input from the cortical and thalamic neurons encoding different sensory and motor variables during behavior and training is an important subject to be further investigated in the future.

\section{Acknowledgements}

We thank Mu-ming Poo, Yong Gu, Yangang Sun and Chengyu Li for valuable discussion; Chunyu Duan and Yu Xin for help in data analysis; Mengjun Sheng and Di Lu for help in surgery; Zhaomei Ying for lab assistant. This work was supported by the National Key R\&D Program of China (grant No. 2017YFA0103900 / 2017YFA0103901); Strategic Priority Research Program of the Chinese Academy of Sciences, Grant No. XDB32010000 
and XDA27010000; NSFC-ISF International Collaboration Research Project, Grant No.

31861143034; Shanghai Municipal Science and Technology Major Project, Grant No. 2018SHZDZX05; the Youth Thousand Talents Plan (to N.L.X.); the National Science Fund for Distinguished Young Scholars (to N.L.X.).

\section{Author contributions}

L.C., S.T. and N.L.X. conceived the project and designed the experiments. L.C. performed the optogenetic experiments. S.T. developed the striatum chronic imaging window method and performed the two-photon imaging experiments. J.P. designed the fiber photometry system. J.P. and N.L.X. developed the behavioral system. K.Z. and B.S. constructed the neural network model and performed the model simulation. Z.Z. performed part of the behavioral training and optogenetic experiments. L.C., S.T. and N.L.X. analyzed the data and wrote the manuscript.

\section{Declaration of interests}

The authors declare no competing financial interests.

\section{Materials \& Correspondence}

Correspondence and requests for materials should be addressed to N.L.X. (xunl@,ion.ac.cn).

\section{Data and materials availability}

All data to understand and assess the conclusions of this study are available in the main text or supplementary materials. All the original behavioral, imaging, histochemical data and analysis code are archived in the Institute of Neuroscience, CAS Center for Excellence in Brain Science and Intelligence Technology, Chinese Academy of Sciences.

\section{References}

1. Albin, R. L., Young, A. B. \& Penney, J. B. The functional anatomy of basal ganglia disorders. Trends Neurosci. 12, 366-375 (1989).

2. Alexander, G. E. \& Crutcher, M. D. Functional architecture of basal ganglia circuits: neural substrates of parallel processing. Trends Neurosci. 13, 266-271 (1990).

3. Cox, J. \& Witten, I. B. Striatal circuits for reward learning and decision-making. Nat. Rev. Neurosci. 20, 482-494 (2019).

4. DeLong, M. R. Primate models of movement disorders of basal ganglia origin. Trends 
Neurosci. 13, 281-285 (1990).

5. Hikosaka, O., Takikawa, Y. \& Kawagoe, R. Role of the Basal Ganglia in the Control of Purposive Saccadic Eye Movements. Physiol. Rev. 80, 953-978 (2000).

6. Klaus, A., Alves da Silva, J. \& Costa, R. M. What, If, and When to Move: Basal Ganglia Circuits and Self-Paced Action Initiation. Annu. Rev. Neurosci. 42, 459-483 (2019).

7. Mink, J. W. THE BASAL GANGLIA: FOCUSED SELECTION AND INHIBITION OF COMPETING MOTOR PROGRAMS. Prog. Neurobiol. 50, 381-425 (1996).

8. Nelson, A. B. \& Kreitzer, A. C. Reassessing Models of Basal Ganglia Function and Dysfunction. Annu. Rev. Neurosci. 37, 117-135 (2014).

9. Hintiryan, H. et al. The mouse cortico-striatal projectome. Nat. Neurosci. 19, 1100-1114 (2016).

10. Hunnicutt, B. J. et al. A comprehensive excitatory input map of the striatum reveals novel functional organization. eLife 5, e19103 (2016).

11. Redgrave, P. et al. Goal-directed and habitual control in the basal ganglia: implications for Parkinson's disease. Nat. Rev. Neurosci. 11, 760-772 (2010).

12. Cui, G. et al. Concurrent activation of striatal direct and indirect pathways during action initiation. Nature 494, 238-242 (2013).

13. Isomura, Y. et al. Reward-Modulated Motor Information in Identified Striatum Neurons. $J$. Neurosci. 33, 10209-10220 (2013).

14. Meng, C. et al. Spectrally Resolved Fiber Photometry for Multi-component Analysis of Brain Circuits. Neuron 98, 707-717.e4 (2018).

15. Sippy, T., Lapray, D., Crochet, S. \& Petersen, C. C. H. Cell-Type-Specific Sensorimotor Processing in Striatal Projection Neurons during Goal-Directed Behavior. Neuron 88, 298-305 (2015).

16. Tecuapetla, F., Matias, S., Dugue, G. P., Mainen, Z. F. \& Costa, R. M. Balanced activity in basal ganglia projection pathways is critical for contraversive movements. Nat. Commun. 5, 4315 (2014).

17. Xin, Y. et al. Sensory-to-Category Transformation via Dynamic Reorganization of Ensemble Structures in Mouse Auditory Cortex. Neuron 103, 909-921.e6 (2019).

18. Zhong, L. et al. Causal contributions of parietal cortex to perceptual decision-making 
during stimulus categorization. Nat. Neurosci. 22, 963 (2019).

19. Hintiryan, H. et al. The mouse cortico-striatal projectome. Nat. Neurosci. 19, 1100-1114 (2016).

20. Znamenskiy, P. \& Zador, A. M. Corticostriatal neurons in auditory cortex drive decisions during auditory discrimination. Nature 497, 482-485 (2013).

21. Xiong, Q., Znamenskiy, P. \& Zador, A. M. Selective corticostriatal plasticity during acquisition of an auditory discrimination task. Nature 521, 348-351 (2015).

22. Klaus, A. et al. The Spatiotemporal Organization of the Striatum Encodes Action Space. Neuron 95, 1171-1180.e7 (2017).

23. Parker, J. G. et al. Diametric neural ensemble dynamics in parkinsonian and dyskinetic states. Nature 557, 177-182 (2018).

24. Bariselli, S., Fobbs, W. C., Creed, M. C. \& Kravitz, A. V. A competitive model for striatal action selection. Brain Res. 1713, 70-79 (2019).

25. Mahn, M. et al. High-efficiency optogenetic silencing with soma-targeted anionconducting channelrhodopsins. Nat. Commun. 9, 1-15 (2018).

26. Burke, D. A., Rotstein, H. G. \& Alvarez, V. A. Striatal Local Circuitry: A New Framework for Lateral Inhibition. Neuron 96, 267-284 (2017).

27. Gittis, A. H., Nelson, A. B., Thwin, M. T., Palop, J. J. \& Kreitzer, A. C. Distinct Roles of GABAergic Interneurons in the Regulation of Striatal Output Pathways. J. Neurosci. 30, 22232234 (2010).

28. Owen, S. F., Berke, J. D. \& Kreitzer, A. C. Fast-Spiking Interneurons Supply Feedforward Control of Bursting, Calcium, and Plasticity for Efficient Learning. Cell 172, 683-695.e15 (2018).

29. Planert, H., Szydlowski, S. N., Hjorth, J. J. J., Grillner, S. \& Silberberg, G. Dynamics of Synaptic Transmission between Fast-Spiking Interneurons and Striatal Projection Neurons of the Direct and Indirect Pathways. J. Neurosci. 30, 3499-3507 (2010).

30. Lo, C.-C. \& Wang, X.-J. Cortico-basal ganglia circuit mechanism for a decision threshold in reaction time tasks. Nat. Neurosci. 9, 956-963 (2006).

31. Howard, C. D., Li, H., Geddes, C. E. \& Jin, X. Dynamic Nigrostriatal Dopamine Biases Action Selection. Neuron 93, 1436-1450.e8 (2017). 
32. Chen, L., Wang, X., Ge, S. \& Xiong, Q. Medial geniculate body and primary auditory cortex differentially contribute to striatal sound representations. Nat. Commun. 10, 1-10 (2019). 33. Guo, L., Walker, W. I., Ponvert, N. D., Penix, P. L. \& Jaramillo, S. Stable representation of sounds in the posterior striatum during flexible auditory decisions. Nat. Commun. 9, 1-10 (2018).

34. Tai, L.-H., Lee, A. M., Benavidez, N., Bonci, A. \& Wilbrecht, L. Transient stimulation of distinct subpopulations of striatal neurons mimics changes in action value. Nat. Neurosci. 15, $1281-1289$ (2012).

35. Yin, H. H. \& Knowlton, B. J. The role of the basal ganglia in habit formation. Nat. Rev. Neurosci. 7, 464-476 (2006).

36. Wang, L., Rangarajan, K. V., Gerfen, C. R. \& Krauzlis, R. J. Activation of Striatal Neurons Causes a Perceptual Decision Bias during Visual Change Detection in Mice. Neuron 97, 13691381.e5 (2018).

37. Kravitz, A. V. et al. Regulation of parkinsonian motor behaviours by optogenetic control of basal ganglia circuitry. Nature 466, 622-626 (2010).

38. Geddes, C. E., Li, H. \& Jin, X. Optogenetic Editing Reveals the Hierarchical Organization of Learned Action Sequences. Cell 174, 32-43.e15 (2018).

39. Tecuapetla, F., Jin, X., Lima, S. Q. \& Costa, R. M. Complementary Contributions of Striatal Projection Pathways to Action Initiation and Execution. Cell 166, 703-715 (2016).

40. Yttri, E. A. \& Dudman, J. T. Opponent and bidirectional control of movement velocity in the basal ganglia. Nature 533, 402-406 (2016).

41. Moss, M. M., Zatka-Haas, P., Harris, K. D., Carandini, M. \& Lak, A. Dopamine Axons in Dorsal Striatum Encode Contralateral Visual Stimuli and Choices. J. Neurosci. 41, 7197-7205 (2021).

42. Shen, W., Flajolet, M., Greengard, P. \& Surmeier, D. J. Dichotomous Dopaminergic Control of Striatal Synaptic Plasticity. Science 321, 848-851 (2008). 


\section{METHODS}

\section{CONTACT FOR REAGENTS AND RESOURCE SHARING}

Further information and requests for resources and reagents should be directed to and will be fulfilled by the Lead Contact, N.L.X. (xunl@ion.ac.cn). This study did not generate new unique reagents.

\section{EXPERIMENTAL MODEL AND SUBJECT DETAILS}

All experimental aspects were carried out in compliance with the procedures approved by the Animal Care and Use Committee of the Institute of Neuroscience, Chinese Academy of Sciences (Center for Excellence in Brain Science and Intelligence Technology, Chinese Academy of Sciences). Male mice, 2- to 3-month-old, D1-Cre (MMRRC_030989-UCD) and D2-Cre (MMRRC_032108-UCD) backcrossed into Black C57BL/6J were used. Male mice, 2- to 3-month-old Black C57BL/6J and PV-Cre mice (Jax stock \#008069) were also used. Mice had no previous history of any other experiments. Mice used for behavioral experiments were housed in a room with a reverse light/dark cycle. On days without behavioral training nor test, the water-restricted mice received $1 \mathrm{~mL}$ of water. On experimental days, mice can receive 0.5 to $1 \mathrm{~mL}$ of water during each behavioral session which lasted for 1 to 2 hours. Each mouse's body weight was measured daily to ensure it was not below $80 \%$ of weight before water restriction.

\section{METHOD DETAILS}

\section{Virus injection for two-photon imaging}

D1-Cre or D2-Cre mice were anesthetized with isoflurane (1 2\%) mixed in air (PinIndexed fill or Funnel-Fill Isoflurane Vaporizer, VETEQUIP), and placed on a stereotaxic frame (RWD 68043 and 68077). Throughout the surgery, body temperature was monitored and maintained at $37^{\circ} \mathrm{C}$ by temperature controller (RWD 69002), and eyes were moisturized with eye ointment. After shaving the hair, the skin was sterilized with $75 \%$ ethanol and iodine. A piece of skin was removed to expose the skull. A small craniotomy (around $0.5 \mathrm{~mm}$ in diameter) was made on the skull vertically above the TS (AP: -1.46, ML: 3.25). The 
stereotactic coordinate for future cannular window implantation was also marked (AP: -1.0, ML: 4.7). Cre-dependent GCaMP6s virus (200-300 nL AAV2/9-hSyn-FLEX-GCaMP6s) was injected slowly with a hydraulic manipulator (Narashige, MO-10) through a glass micropipette (pulled using Sutter Instrument P-97, with tip broken into an opening with 20-30 $\mu \mathrm{m}$ inner diameter; Drummond Scientific, Wiretrol II Capillary Microdispenser) into the TS at a depth of $-3.4 \mathrm{~mm}$ from the level of bregma.

Following the injection, the micropipette was left in the injection site for $\sim 10 \mathrm{~min}$ and then retracted slowly to reduce flow back of the viruses. The craniotomy was then covered with low-toxicity silicon adhesive (Kwik-Sil, WPI). After covering the exposed skull and surrounding tissues with instant adhesive (LOCTITE 495, Henkel), a custom-designed titanium headplate was attached to the skull with dental cement. After surgery, mice were allowed to recover with full access to water for at least 7 days. Virus preparations were obtained from Shanghai Taitool Bioscience Co.Ltd, or OBiO Technology (Shanghai) Corp., Ltd.

\section{Behavioral training}

After one-week recovery following surgery, mice were restricted with water for $\sim 7$ days, while $1 \mathrm{~mL}$ water was given to each mouse per day. After water restriction, behavior training was started. Mice performed one behavioral training session each day, and were allowed to perform the task until sated. The mice's body weights were measured and water consumption was calculated using the body weight change before and after each session. If mice consumed less than $0.5 \mathrm{~mL}$ water per day, additional water supplement was provided.

The behavioral apparatus has been described previously ${ }^{1,2}$. Briefly, experiments were conducted inside custom-designed double-walled sound-attenuating boxes. The mouse auditory decision behavior was controlled by a custom-developed PX-Behavior System. Cosine ramps $(5 \mathrm{~ms})$ were applied to the rising and falling of all tone stimuli. The sound waveforms are amplified using ZB1PS (Tucker-Davis Technologies) and delivered by an electrostatic speaker (ES1, Tucker-Davis Technologies) placed on the right side of the mice. The sound system was calibrated using a free-field microphone (Type 4939, Brüel and Kjær) over 3-60 kHz to ensure a flat spectrum ( $\pm 5 \mathrm{~dB}$ SPL). Behavioral trials were synchronized with two-photon image acquisition by digital outputs from the PX-Behavior System to the 'ScanImage' software which controls the two-photon imaging setup. In optogenetics experiments, the lasers were also controlled through digital outputs from PX-Behavior System. 
The behavioral task is based on the auditory-guided two-alternative-forced-choice (2AFC) task on head-fixed mice as described before ${ }^{1,2}$. After mice learnt how to lick left and right water ports to get water, they were trained to discriminate a range of tone frequencies (between 7 and $28 \mathrm{kHz}$, or between 5 and $20 \mathrm{kHz}$, distributed in logarithmic scale of octaves) as higher or lower than the defined category boundary, which is the mid line of the logarithmically spaced frequency range, i.e., $14 \mathrm{kHz}$ for the $7-28 \mathrm{kHz}$ range, and $10 \mathrm{kHz}$ for the 5-20 kHz. The frequency ranges were chosen according to the typical hearing range of laboratory mouse ${ }^{3}$. Sound intensity is $70 \mathrm{~dB}$ SPL for all used frequencies. The initiation of each trial is not explicitly cued, and the animal needs to wait for the tone stimulus to occur. Following the inter-trial interval (ITI), a $0.5-1 \mathrm{~s}$ random delay was imposed before tone stimuli (lasting for $300 \mathrm{~ms}$ ) to ensure that animals cannot predict the onset of tone stimuli. Mice were required to respond by licking left or right water port within a $3 \mathrm{~s}$ answer period following the stimulus offset to report their perceptual judgements of the tone as the high frequency or low frequency categories. To control for systematic side bias, some mice were trained to report high frequencies by licking right port and low frequencies the left port (lowleft / high-right; Extended Data Fig. 7b), while others were trained with the reversed association (low-right / high-left; Extended Data Fig. 7c). Correct responses led to opening of the water valve for a short period of time to dispense a small amount of water reward $(\sim 1.5$ $\mu \mathrm{L})$. Error responses lead to a $2 \sim 6 \mathrm{~s}$ time-out punishment, during which licking to the wrong side would reinitiate the time-out period. Mice performed one behavioral training session each day, and were allowed to perform the task until sated. Mice completed about 500 trials in each training session and the trial numbers varied among animals.

Mice were first trained with the two easy frequencies separated by 2 octaves, which are the end frequencies of the tested ranges, e.g., 7 and $28 \mathrm{kHz}$, or 5 and $20 \mathrm{kHz}$. Mice need about 10 training sessions to learn the task rule, and the learning rate varied among animals. After the correct rate reached criteria $85 \%, 6$ tone stimuli of intermediate frequencies (between 7 and $28 \mathrm{kHz}$ or 5 and $20 \mathrm{kHz}$, linearly spaced on the logarithmic scale) were delivered in randomly interleaved trials. For each session, the fraction of trials with tones of the intermediate frequencies is $\sim 30 \%$ to keep the performance stable.

To quantify the proportion of contralateral choices in optogenetic manipulation and in imaging experiments, we pool all animals trained with either of the two types of associations ('low-left / high-right' or 'low-right / high-left') together by ordering the tone frequencies according to relative octaves ranging from -1 to 1 , with 0 at the boundary frequency, the negative values representing frequencies associated with the ipsilateral choices and positive 
values the contralateral choices.

Psychometric function was obtained by fitting the behavioral data using a 4-parameter sigmoidal function 4,5

$$
y(x)=g+(1-g-l) * 0.5 *\left(1+\operatorname{erf}\left((x-u) / \operatorname{sqrt}\left(2 * v^{\wedge} 2\right)\right)\right)
$$

where $y(x)$ is the probability that animal would make a right choice (Fig. 1c) or a contralateral choice (Fig. 5, 6e, and Extended Data Fig. 7), and $x$ is the tone frequency (in octave). Parameters to be fitted are: $g$ the guess rate, $l$ the lapse rate, $u$ the subject bias (boundary), and $v$ the discrimination sensitivity (threshold). erf () represents error function. For psychometric function fitting (Fig. 1c, Fig. 5, 6E and Extended Data Fig. 7), 'miss' trials in which mice did not response during the answer period were excluded.

\section{Cannula window implantation for two-photon calcium imaging}

When the behavioral performance reached the criterion of $85 \%$ correct, mice were allowed to have freely access to water for 2-3 days before the surgery of implanting cannular imaging window. During the surgery, mice were anaesthetized with isoflurane (1 2\%), and an $\sim 2 \mathrm{~mm}$ diameter craniotomy was made centered at the marker for window implantation. The tissue above the TS were removed by aspiration through 25-gauge and 27-gauge blunt needles connected to a peristaltic pump (LongerPump YZ1515x). During the aspiration, saline was continuously delivered, and moisturized gelatin sponge (Xiang'en) was applied to prevent the blood from clotting on the striatum surface. A small amount of uncured Kwik-sil was applied to the striatum surface and a custom-made cannula window (1.8 $\mathrm{mm}$ in outer diameter, $1.4 \mathrm{~mm}$ in depth, Extended Data Fig. 1a) was carefully lowered into the brain. The outer ring of the cannula window was then fixed to the skull with instant adhesive (LOCTITE 495, Henkel) and dental cement. The inside of the cannula was then filled with Kwik-sil as a protector. After surgery, mice were allowed to recover with full access to water for at least 7 days before behavior training was resumed.

\section{Two-photon calcium imaging}

Calcium imaging was performed using a custom built two-photon microscope (https://wiki.janelia.org/wiki/display/shareddesigns/MIMMS). The entire microscope was enclosed in a custom-designed, double-walled sound-attenuating box to eliminate potential influence from ambient noise of the two-photon imaging system (internal noise level $<30 \mathrm{~dB}$ SPL with the two-photon imaging system running). The noise produced by the resonant scanner were attenuated to $<30 \mathrm{~dB}$ SPL by an optical window sealing the output opening of 
the resonant scanner module. GCaMP6s was excited using a Ti-Sapphire laser (Chameleon UltraII, Coherent) tuned to $925 \mathrm{~nm}$. Images were acquired using a 16X 0.8 NA objective (Nikon), and the GCaMP6s fluorescence was isolated using a bandpass filter (525/50, Semrock), and detected using GaAsP photomultiplier tubes (10770PB-40, Hamamatsu). Horizontal scanning was accomplished using a resonant galvanometer (Thorlabs; $16 \mathrm{kHz}$ line rate, bidirectional). The average power for imaging was $\sim 70 \mathrm{~mW}$, measured at the entrance pupil of the objective. The field of view was $\sim 500 \times 500 \mu \mathrm{m}$ (at zoom $2 \times$ ) or $\sim 350 \times 350 \mu \mathrm{m}$ (at zoom $3 \times$ ) imaged at frame rate of $\sim 28 \mathrm{~Hz}$ for $512 \times 512$ pixels (Bi-direction) or $256 \times 512$ pixels (Single-direction). The acquisition system was controlled by ScanImage (https://scanimage.org) ${ }^{6}$. For each mouse, the optical axis was adjusted (40 50 degree from vertical) to be perpendicular to the imaging window. Different fields of view in the same mouse were imaged on different sessions. The imaging was acquired continuously within each session, while the ScanImage system received a tag signal from the PX-Behavior System at the initiation of each trial (after ITI).

\section{Surgical procedures for fiber photometry and optogenetics}

To prepare animals for fiber photometry recordings or optogenetic manipulations, mice were anaesthetized with isoflurane (1 2\%), and a small craniotomy ( $400 \mu \mathrm{m}$ in diameter) was made vertically above the striatum in each hemisphere. For fiber photometry recording from dSPNs axons in SNr, $300 \mathrm{~nL}$ AAV2/9-hSyn-FLEX-GCaMP6s was slowly injected in bilateral TS (AP: -1.46, ML: 3.25, DV: -3.4) of D1-Cre mice, and fiber optics (NA 0.37, 200 $\mu \mathrm{m}$ core, ferrule O.D., $1.25 \mathrm{~mm}$ ) were implanted into the bilateral SNr (AP: -3.5, ML: 1.8, DV: 4.3) (Extended Data Fig. 8a). For fiber photometry recording from the PV neurons, 300 nL AAV2/9-hSyn-FLEX-GCaMP6s was injected into bilateral TS of PV-Cre mice, and fiber optics (NA 0.37, $200 \mu \mathrm{m}$ core, ferrule O.D. 1.25mm) were implanted $\sim 200 \mu \mathrm{m}$ above the virus injection sites (Extended Data Fig. 8b). After implanting the fiber optics, silicon adhesive (Kwik-sil, WPI) was used to cover the craniotomy. A custom-designed titanium headplate was attached to the skull with dental cement for head fixation. After surgery, mice were allowed to recover at least 7 days before water restriction and behavioral training.

For optogenetic manipulations of specific types of striatal neurons, $400 \mathrm{~nL}$ AAV2/8CAG-FLEX-ChR2(H134R)-mCherry (for activation, Extended Data Fig. 8c, $2.58 \times 10^{13}$ V.G./mL) or $400 \mathrm{~nL}$ AAV2/9-Ef1 $\alpha$-DIO-eNpHR3.0-eYFP (for inactivation, Extended Data Fig. $8 \mathrm{~d}, 1.50 \times 10^{13}$ V.G. $/ \mathrm{mL}$ ) virus was slowly injected into bilateral TS in D1-Cre or D2-Cre transgenic mice. About 30 min after virus injection, fiber optics (NA 0.37, $200 \mu \mathrm{m}$ core, 
ceram ferrule OD $1.25 \mathrm{~mm}$ ) were implanted $\sim 200 \mu \mathrm{m}$ above the virus injection sites. For nonselective inactivation of striatal neurons, $400 \mathrm{~nL}$ AAV2/9-CAG-ArchT-GFP $\left(3.50 \times 10^{12}\right.$ V.G./mL) virus was injected bilaterally to TS or to an anterior sub-region of dorsal stratum (AP: -0.02, ML: 2.0, DV: -3.4) in wildtype C57BL/6J mice (Extended Data Fig. 8e). For control animals, AAV2/9-CAG-eGFP-2A $\left(2.40 \times 10^{13}\right.$ V.G./mL $)$ virus was injected into the bilateral TS followed by implantation of fiber optics (Extended Data Fig. 8f).

\section{Fiber photometry recording}

The fiber photometry system was custom built based on previous method ${ }^{7}$. The excitation light was from a 470-nm LED (Cree, Inc, XQEBLU-SB-0000-00000000Y01-ND) and a 410-nm LED (Xuming, 3W, 410-420 nm). The intensity of the 470-nm light was set to 10-15 $\mu \mathrm{W}$ measured at the distal end of the patch cord (calibrated based on the transmittance of the fiber optics implanted in the brain, to keep the intensity at $10 \mu \mathrm{W}$ around the fiber optic tips). For each recording site, the intensity of 410-nm light was adjusted to keep the recorded emission fluorescence level excited by 410-nm light close to that excited by 470-nm light from the brain tissue.

The lights from 470-nm LED and 410-nm LED independently passing through aspheric condenser lenses (Thorlabs, ACL25416), were filtered through respective bandpass filters (FB470-10, and FB410-10, Thorlabs), and were then combined by a dichroic mirror (DMLP425R, Thorlabs). The combined excitation lights then sequentially passed through a dichroic mirror (Chroma, T525/50dcrb) and an objective (Olympus, UPLFLN 20X NA0.5), and then were coupled into a fiber optic cable (NA 0.37, $200 \mu \mathrm{m}$ core) that affixed to the fiber optics implanted in mice's brain. A small fraction of 470-nm and 410-nm lights reflected by the first dichroic was collected by a condenser lens (Thorlabs, ACL25416) and received by a photodiode (Thorlabs, PAD100A2), the real-time light intensity of which was used as a feedback signal for custom-designed closed-loop circuits to stabilize the intensity of 470-nm and 410-nm lights throughout the recording session (Extended Data Fig. 4a).

The emitted fluorescent signals were collected by the same light path and focused onto a CCD (QImaging, R1 retina). The 470-nm LED, the 410-nm LED and the CCD were controlled by a NI I/O device (NI, USB-6341) and software LabVIEW. The NI device also received the triggers from the digital outputs of PX-Behavior System to tag the behaviorrelated time. Consecutive camera frames were captured at $40 \mathrm{~Hz}$ using alternating $470-\mathrm{nm}$ and 410-nm excitation lights within each session, so each of the 470-nm and 410-nm channels was sampled at $20 \mathrm{~Hz}$. The excitation pulse duration of 470-nm LED or 410-nm 
LED in each frame was $11 \mathrm{~ms}$ for an exposure time of $10 \mathrm{~ms}$, beginning at $0.5 \mathrm{~ms}$ prior to the onset of the exposure time of CCD and ending at $0.5 \mathrm{~ms}$ after the offset of the exposure time of CCD. The signals from CCD were recorded by ImageJ based software Micro-Manager (Version 2.0 beta), with the 'binning' at 16x16, and the 'Exposure' time of 10ms. The fluorescent signals excited by the 470-nm and 410-nm were extracted and analyzed subsequently using MATLAB scripts.

\section{Optogenetic manipulations}

For cell-type specific activation by ChR2 (Fig. 5a-5h), AAV-CAG-FLEX-ChR2mCherry was injected into the TS of D1-Cre or D2-Cre mice to express ChR2 in Credependent manner in dSPNs or iSPNs. A 473-nm diode-pumped solid state laser (BL473T8100FC, Shanghai Laser \& Optics Century Co., Ltd.) with the output power adjusted to $\sim 2$ $\mathrm{mW}$ at the distal end of patch cable was used to activate ChR2-expressing neurons. To inactivate dSPNs, iSPNs or PV neurons (Fig. 5i-51 and 6d-6e), NpHR3.0 was expressed using AAV2/9-Ef1 $\alpha$-DIO-eNpHR3.0-eYFP. A 593-nm diode-pumped solid state laser (YL593T6100FC, Shanghai Laser \& Optics Century Co., Ltd.) with output power of $\sim 20 \mathrm{~mW}$ at the distal end of patch cable was used to inactivate NpHR-expressing neurons. For iSPN inactivation by stGtACR2 (Fig. 5m and 5n), AAV2/9-hSyn-SIO-stGtACR2-FusionRed was injected into the TS of D2-Cre mice, and a 462-nm blue diode laser (BLM462TA-300FC, Shanghai Laser \& Optics Century Co., Ltd.) with an output power of $2 \mathrm{~mW}$ at the distal end of patch cable was used to inactivate stGtACR2-expressing iSPNs.

For inactivation experiments using ArchT (Extended Data Fig. 7), AAV2/9-CAG-ArchTGFP was injected into the testing group, and AAV2/9-CAG-eGFP-2A was injected to the control group. A 532-nm diode-pumped solid state laser (GL532TA-100FC, Shanghai Laser \& Optics Century Co., Ltd.) with an output power of $20 \mathrm{~mW}$ at the distal end of patch cable was used.

In all optogenetic stimulation sessions, photostimulation was applied to partial of the trials, which were randomly interleaved with control trials (no photostimulation) to achieve within-subject and within-session comparison. Since in most sessions there were less trials with intermediate frequencies $(\sim 30 \%)$ and more trials with the end frequencies $(\sim 70 \%)$, to obtain comparable number of trials with photostimulation for intermediate and end frequencies, photostimulation was applied to $\sim 50 \%$ of the intermediate-frequency trials, and $\sim 15 \%$ of end-frequency trials. For photostimulation trials in Fig. 5a-5d, 5i-5n, 6d-6e and Extended Data Fig. 7, $1 \mathrm{~s}$ of continuous laser pulse was delivered unilaterally starting from 
the tone stimuli onset. For sub-trial activation of iSPNs, $150 \mathrm{~ms}$ of continuous laser were used within the second half of the sound stimulus period (Fig. 5h) or starting $650 \mathrm{~ms}$ before the sound stimulus onset (Fig. 5g). In Fig. 5f, photostimulation was within a $150 \mathrm{~ms}$ window after sound stimulus onset (4/9 sessions) or within a $200 \mathrm{~ms}$ window starting from $50 \mathrm{~ms}$ before sound stimulus onset (5/9 sessions).

\section{Histological Verification}

After the completion of experiments, mice were deeply anesthetized and perfused with saline and 4\% PFA (or 10\% formalin), and brains were removed and post-fixed overnight. Brains were then incubated with PBS containing 30\% sucrose overnight. Frozen sections (50 $\mu \mathrm{m}$, using Leica CM1950 cryostats) containing viral injection sites, cannula window location and optic fiber tracks were collected and stained with DAPI to visualize cell nuclei. Histology images were taken using Olympus VS120® Virtual Microscopy (Extended Data Fig. 8). For verification of cell-type specific virus expression in dSPNs and iSPNs, $200 \mathrm{~nL}$ AAV2/8CAG-FLEX-eGFP-WPRE-pA (1.25×1013 V.G./mL, TaiTool Bioscience) was injected into the TS of D1-Cre or D2-Cre. One month after the surgery, mice's brains were sectioned sagittally and the projection pattern of neurons expressing GFP were checked (Extended Data Fig. $1 \mathrm{~b}$ and $1 \mathrm{c})$.

\section{QUANTIFICATION AND STATISTICAL ANALYSIS}

\section{Two-photon imaging data analysis}

All imaging data analysis was performed by custom-written MATLAB (Mathworks) routines. Within each session, the brain motion artifacts were corrected by registering each frame to a target image using a cross-correlation-based registration algorithm (discrete Fourier transformation (DFT) algorithm $)^{8}$. The target image was the averaged projection of several visually identified frames with minimal brain motion artifacts. We identified individual neuron as a region of interest (ROI) based on its morphological properties using the averaged projection of images from each trial. The average fluorescence values of all pixels within each ROI across frames in one session were used as the raw fluorescence data $\left(\mathrm{F}_{\text {raw }}\right)$ of each neuron. The slow fluorescence change was removed by subtracting the $8^{\text {th }}$ percentile value of $F_{\text {raw }}$ within nearby 500 frames for each frame (forward 250 frames and backward 250 frames, $\sim 17.5 \mathrm{~s}$ ). The mode of all these $8^{\text {th }}$ percentile values within one session was then added back to restore the fluorescence levels of each neuron, resulting in the 
corrected fluorescence data $(\mathrm{F})$. The calcium signal $(\Delta \mathrm{F} / \mathrm{F})$ was calculated as:

$$
\Delta F / F=\left(F-F_{0}\right) / F_{0} \times 100 \%
$$

Where $\mathrm{F}_{0}$ is the baseline fluorescence defined as the middle value of the peak histogram of F (bin size, 500). Estimation of spike rate using calcium signal deconvolution was based on the Fast Nonnegative Deconvolution algorithm ${ }^{9}$ (Fig. 2i-2p and 2r).

To quantify single neuronal selectivity differentiating trial types with ipsilateral and contralateral choices, we used an ideal observer decoding based on ROC analysis ${ }^{10}$. By comparing the distribution of the neuronal responses in contralateral choice trials and those in ipsilateral trials (with respect to the imaged hemisphere), the area under ROC curve (auROC) value for choice quantifies how well a neuron could differentiate between contralateral choice and ipsilateral choice. We then calculated neurons' relative preference for the contralateral vs ipsilateral trials using selectivity index (SI) by rescaling the auROC value to range $(-1,1)$ as following:

$$
S I=2 \times(\text { auROC }-0.5)
$$

where value 1 indicates the strongest preference for contralateral choices and value -1 for strongest ipsilateral choices ${ }^{11}$.

The significance level of SI was defined by a permutation analysis, where we shuffled the trial label (contralateral or ipsilateral choice) for 1000 times and each time we calculated an SI. Then values greater than 99.5 th percentile or smaller than 0.5 th percentile of the shuffled results are considered as $\mathrm{p}<0.01$. The SIs for visualization (Extended Data Fig. 2) were acquired by a generalized ROC algorithm ${ }^{12}$ with a 1-frame-per-step and 6-frame-width backward sliding window. The SI values used for statistical analysis (Fig. 2a, 2c, 2i, 2k) were obtained using the MATLAB function (rocOnline.m in MATLAB). To calculate the SI, mean $\Delta \mathrm{F} / \mathrm{F}$ of each trial during $100 \mathrm{~ms}$ preceding mice answer time was used in Fig. 2a-2d, and mean inferred spike rate during $100 \mathrm{~ms}$ preceding mice answer time was used in Fig. 2i-21. Contralateral-preferring neurons: with significant contralateral-preference in correct and error trials; ipsilateral-preferring neurons: with significant ipsilateral-preference in correct and error trials; sensory category preference: with opposite significant preference in correct and error trials (Fig. 2a, 2c, 2i, 2k; Fig. 3h-3k ). Neurons with significant contralateral-preference in correct trials and neurons with significant ipsilateral-preference in correct trials were included in Fig. 2b, 2d, 2j and 21. Only SI in correct trials (Extended Data Fig. 2) are used.

To quantify the temporal correlation between two simultaneously imaged neurons, we calculated the Pearson's correlation coefficient (CC) between the two time series of calcium 
signals (Fig. 3). Averaged CCs between neuronal activities were reported as the average across neuronal pairs (Fig. 3c). In Fig. 3e-3g and Extended Data Fig. 3, all neurons in the imaging field are used to calculate CCs. In Fig. 3h-3k, only neurons with significant choiceselectivity were included. The cross-correlation between neuronal pairs were obtained by compute the Pearson's correlation coefficients between the $\Delta \mathrm{F} / \mathrm{F}$ traces from the two neurons with different time lags, ranging from $-10 \mathrm{~s}$ to $+10 \mathrm{~s}$, which were then plotted as a function of the time lag (Fig. 3c and 3d). We do not exclude the possibility that mixing of optical signals from neuropils, which cannot be optically isolated, could potentially contribute to the level of correlation ${ }^{13}$. However, for dSPNs or iSPNs, the neuropils are all from the genetically defined cell types, thus the cell-type specific difference in the correlation coefficients still reflects the difference in the level of local population coordination between dSPNs and iSPNs.

\section{Fiber photometry data analysis}

The 410-nm reference trace was scaled to best fit the 470-nm signals using least-squares regression ${ }^{14}$. Then the motion-corrected $470-\mathrm{nm}$ signal $\left(\mathrm{F}_{470}\right)$ was obtained by subtracting the scaled 410-nm reference trace from the 470-nm signal and then adding the mean value of scaled $410-\mathrm{nm}$ trace. $\Delta \mathrm{F} / \mathrm{F}$ signal was calculated by subtracting the mode of $\mathrm{F}_{470}$ and then dividing by the mode value of $\mathrm{F}_{470}$.

To compare signals across recording sessions, we used the z-score of calcium signals, which is the $\Delta \mathrm{F} / \mathrm{F}$ subtracted by the mean and divided by the standard deviation of the data in the session. The peak value of z-score within $1 \mathrm{~s}$ after mice's answer time was used for comparison (Fig. 4c, 6c).

To quantify the encoding of mice's choice direction (Contra. vs Ipsi. choice) in the neuronal activity measured with fiber photometry, mean $\Delta \mathrm{F} / \mathrm{F}$ of each trial during $1 \mathrm{~s}$ after mice's answer time was used to calculate the selectivity index (SI) in each recording session. Statistical significance was determined with a permutation test at the significance level of $\alpha=$ 0.05 (Fig. 4d).

\section{Optogenetics data analysis}

For analyzing the behavioral effects of optogenetic stimulation, 'miss' trials were excluded. To quantify the behavioral effects of optogenetic stimulation, we used the two-way repeated-measures ANOVA with function 'fitrm' in Matlab to test the effect across tone frequencies, and use LSD post hoc tests to compare the effects at each frequency. 


\section{Statistical Analysis}

All statistical analysis was performed using MATLAB 2017a (MathWorks). All data are shown as mean \pm s.e.m. and shaded regions surrounding line-plots indicate s.e.m. unless mentioned otherwise. The comparison significance was tested using KolmogorovSmirnov test, one-sample proportion test, paired two-sided Wilcoxon signed rank test, twoway repeated-measures ANOVA and post hoc Fisher's LSD multiple comparison tests. $* \mathrm{P}<$ $0.05, * * \mathrm{P}<0.01, * * * \mathrm{P}<0.001$. For fiber photometry recording from $\mathrm{SNr}$, two sessions were excluded due to the inaccuracy of fiber optics tip location. For activation of iSPNs, one session was excluded due to inconsistent sound frequency setting with other sessions.

\section{NEURAL NETWORK MODEL}

To examine the potential computational implementation for how concurrent but asymmetrical dSPN and iSPN activity could result in unambiguous action selection following decisions, we constructed a computational model of the cortico-basal ganglia circuitry on the population level, which is similar to the computational model of dopamine-biasing action selection through basal ganglia in the previous study ${ }^{15}$. In the model, the decision outcomes are transferred from cortex to dSPNs, iSPNs and striatal PV neurons via canonical corticostriatal projections ${ }^{16}$. The activity of the dSPNs and iSPNs populations converge to the ipsilateral substantia nigra pars reticulata $(\mathrm{SNr})$ populations through the direct and the indirect pathway respectively. The left and right SNr populations mutually inhibit each other, and the dominant $\mathrm{SNr}$ population determines the motor output by controlling the final motor output either through brainstem circuits or motor cortices. The network model composed of neural populations in both left and right hemispheres with symmetrical connectivity, as shown in Fig. 8a.

In the model, a perceptual decision $d$ is produced in the cortex according to a simple decision rule (Equation 1). Where $\operatorname{sign}(q)=1$ if $q \geq 0, \operatorname{sign}(q)=1$ if $q<0$. Note that the tone of stimulus $q \in[-1,1]$ is in the unit of octaves. $\theta(q)$ is the probability of wrong decision due to perceptual uncertainty, where $\sigma=2.24$ is the degree of uncertainty in the perception (Equation 2). The decision outcome $d= \pm 1$ encodes left or right choice. The cortical neurons in each hemispace are organized into two types of populations that are selective to contralateral or ipsilateral choices. The firing rate of cortical population $C T X_{j k}$ is shown in Equation 3, where $j \in\{L, R\}$ representing the left or right hemisphere, $k \in$ 
\{contra, ipsi\} is the selectivity for left $(s=-1)$ or right $(s=1)$ action, $s=-1$ corresponds to $f^{C T X_{\text {Rcontra }}}$ and $f^{C T X_{\text {Lipsi }}}, s=1$ corresponds to $f^{C T X_{\text {Lcontra }}}$ and $f^{C T X_{R i p s i}}$, and $d$ is the decision outcome given by Equation 1. $I=20$ is the peak firing rate of cortical neurons. $f_{b}=0$ is reference frequency. As the frequency $q$ changes, the firing activity of cortical neural population switches between low and high activity states in a sigmoid form, with the switching slope controlled by $\zeta=0.01$. Cortical neurons keep selfsustained activity states for a period of time without external input ${ }^{17}$.

$$
\begin{gathered}
d=\left\{\begin{array}{c}
-\operatorname{sign}(q), \text { with probality } \theta(q) \\
\operatorname{sign}(q), \text { with probality } 1-\theta(q)
\end{array}\right. \\
\theta(q)=\frac{1}{2} \exp \left(-\frac{q^{2}}{\sigma^{2}}\right) \\
f^{C T X_{j k}}(q, d)=\frac{I}{1+\exp \left(\frac{d s\left(q-f_{b}\right)}{\zeta}\right)}
\end{gathered}
$$

SPNs in the striatum receive currents both externally and internally (Equation 4), where $I_{\text {ext }}^{\alpha}$ and $I_{i n t}^{\alpha}$ are the external current and internal current to population $\alpha$, from the cortex and from striatal sub-populations respectively (Fig. 8a). SPNs are divided into 8 subpopulations based on different response preferences found in our imaging results and from the two hemispheres, $\alpha=D_{i j k}$, with $i \in\{1,2\}$ representing cell types (dSPNs and iSPNs). The cortical input to population $\alpha$ is shown in Equation 5, where $f^{C T X_{j k}}$ is the activity of the cortical population with selectivity $s$ (Equation 3 ). $w_{\alpha}^{\beta}$ is the connection strength from population $\beta$ to population $\alpha$ (Table S1). $V_{\alpha}$ is the membrane potential. For the excitatory connection from $C T X_{j k}$, the reverse potential $E_{C T X_{j k} \rightarrow \alpha}=0$. The internal input current to population $\alpha$ is shown in Equation 6, where $\beta$ is a striatal population including $\alpha$ and $\mathrm{PV}$, and $f^{\beta}$ is its firing activity. Reverse potential parameters are set according to precious study ${ }^{18}$. For inhibitory connections from striatal population $\beta$, the reverse potential was $E_{\beta \rightarrow \alpha}=$ $-65 m V$. For the connection from D2 to SNr, $E_{D_{2 j k} \rightarrow S N r_{j}}=0 \mathrm{mV}$.

$$
\begin{gathered}
I^{\alpha}=I_{e x t}^{\alpha}+I_{i n t}^{\alpha} \\
I_{e x t}^{\alpha}=\sum_{S= \pm 1} W_{\alpha}^{C T X_{j k}}\left(E_{C T X_{j k} \rightarrow \alpha}-V_{\alpha}\right) f^{C T X_{j k}}(q, d) \\
I_{\text {int }}^{\alpha}=\sum_{\beta} w_{\alpha}^{\beta}\left(E_{\beta \rightarrow \alpha}-V_{\alpha}\right) f^{\beta}(t)
\end{gathered}
$$

Take $D_{1, L, \text { Contra }}$ as an example of SPN sub-population to explain its specific input current shown in Equation 4-6,

$$
I^{D_{1, L, \text { Contra }}}=I_{\text {ext }}^{D_{1, L, \text { Contra }}}+I_{\text {int }}^{D_{1, L, \text { Contra }}}
$$




$$
\begin{gathered}
I_{\text {ext }}^{D_{1, L, \text { Contra }}}=\sum_{S= \pm 1} w_{D_{1, L, \text { Contra }}}^{C T X_{S}}\left(E_{C T X_{S} \rightarrow D_{1, L, \text { Contra }}}-V_{D_{1, L, \text { Contra }}}\right) f^{C T X_{S}}(q, d) \\
I_{\text {int }}^{D_{1, L, \text { Contra }}}=\sum_{\beta} W_{D 1, L, \text { Contra }}^{\beta}\left(E_{\beta \rightarrow D_{1, L, \text { Contra }}}-V_{D_{1, L, \text { Contra }}}\right) f^{\beta}(t)
\end{gathered}
$$

PV neurons only receive cortical inputs from ipsilateral cortex, $I^{P V_{j}}=I_{e x t}^{P V_{j}}, j \in\{L, R\}$, take PV neurons in the left hemisphere as an example,

$$
I^{P V_{L}}=w_{P V_{L}}^{C T X_{L k}}\left(E_{C T X_{L k} \rightarrow P V_{L}}-V_{P V_{L}}\right) f^{C T X_{L k}}(q, d) .
$$

SNr neurons receive external current from SPNs and internal current as their inputs.

Take SNr neurons in the left hemisphere as an example,

$$
\begin{gathered}
I^{S N r_{L}}=I_{\text {ext }}^{S N r_{L}}+I_{\text {int }}^{S N r_{L}} \\
I_{\text {ext }}^{S N r_{L}}=\sum_{\alpha} w_{S N r_{L}}^{\alpha}\left(E_{\alpha \rightarrow S N r_{L}}-V_{S N r_{L}}\right) f^{\alpha}(t) \\
I_{i n t}^{S N r_{L}}=w_{S N r_{L}}^{S N r_{L}}\left(E_{S N r_{L} \rightarrow S N r_{L}}-V_{S N r_{L}}\right) f^{S N r_{L}}(t)+w_{S N r_{L}}^{S N r_{R}}\left(E_{S N r_{R} \rightarrow S N r_{L}}-V_{S N r_{L}}\right) f^{S N r_{R}(t)}
\end{gathered}
$$

The membrane potential of a striatal population $\alpha$ is described as shown in Equation 7. $E_{\alpha}=-55 \mathrm{mV}$ is the reversal potential. $\epsilon$ is a noise term. The firing frequency of a striatal population $\alpha$ is shown in Equation 8. The transfer function $g$ is linear above the discharge threshold $\theta=-40 \mathrm{mV}$ and has an exponential growth trend below the discharge threshold (Equation 9), where $k=1$ represents medium spiny neurons, and $k=5$ represents interneurons in the striatum.

$$
\begin{gathered}
\tau \frac{d V^{\alpha}}{d t}=E_{\alpha}-V^{\alpha}+I^{\alpha}+\epsilon \\
f^{\alpha}(t)=g\left(V^{\alpha}-\theta\right) \\
g(x)=k \log (1+\exp (x))
\end{gathered}
$$

The motor choice is made according to the $\mathrm{SNr}$ activity after $100 \mathrm{~ms}$ when the network activity becomes stabilized. The rule for motor choice is defined in Equation 10.

$$
\text { Motor choice }=\left\{\begin{array}{l}
\text { Left, } \quad f^{S N_{r_{L}}-f^{S N_{r_{R}}} \geq 0} \\
\text { Right, } f^{S N_{r_{L}}-f^{S N_{r_{R}}}<0}
\end{array}\right.
$$

The connection weights between the populations in the network are shown in Table S1. The weights are assumed to form through pervious learning processes. Populations are labeled according to the convention introduced in previous sections. For example, CTX ${ }_{-1}$ is the cortical population selective to left choice, and $D_{1 \text { Rcontra }}$ is the D1 population connected to the right $\mathrm{SNr}$ and is selective for contralateral choice. For simplicity, we omitted some 
indices of the labels if they are not concerned. For example, $D_{1 L}$ includes populations $D_{1 \text { Lipsi }}$ and $D_{1 \text { ccontra }}$. The dSPNs and iSPNs in the striatum inhibit each other, and the weights are shown as $w_{D i^{\prime} j k^{\prime}}^{D i j k}$.

The optogenetic manipulation of a striatal population $\alpha$ is modelled by an additional current $I^{\text {stim }}$ to the membrane potential dynamics (Equation 11), where $I^{\text {stim }}$ is the regulation current, taking positive or negative values for optogenetic activation or inactivation, respectively. The parameters of optogenetic regulation are shown in Table $\mathbf{S 2}$.

$$
\tau \frac{d V^{\alpha}}{d t}=E_{\alpha}-V^{\alpha}+I^{\alpha}+I^{\text {stim }}+\epsilon
$$

We performed the network model stimulation using the same set of parameters under the standard control conditions (Table S1), as well as under the perturbed conditions caused by optogenetic activation or inactivation (Table S2). Under each condition, 300 independent trials were simulated, and the probability and the standard deviation of choosing right choice was calculated. Psychometric functions were fitted by a 4-parameter sigmoidal curve same as in the experimental data. Compared with the standard control situation, under the perturbed condition of optogenetic activation/inactivation of striatal PV and D1-/D2-SPNs, the model reproduced similar biased action selection behavior as observed under the corresponding conditions in experiments (Fig. 8d-8h). The psychometric functions of action selection of the model qualitatively captured the deviation from that of the control condition after perturbation as shown in experiments.

\section{SUPPLEMENTAL REFERENCES}

1. Xin, Y. et al. Sensory-to-Category Transformation via Dynamic Reorganization of Ensemble Structures in Mouse Auditory Cortex. Neuron 103, 909-921.e6 (2019).

2. Zhong, L. et al. Causal contributions of parietal cortex to perceptual decision-making during stimulus categorization. Nat. Neurosci. 22, 963 (2019).

3. Heffner, H. E. \& Heffner, R. S. Hearing ranges of laboratory animals. J. Am. Assoc. Lab. Anim. Sci. JAALAS 46, 20-22 (2007).

4. Carandini, M. \& Churchland, A. K. Probing perceptual decisions in rodents. Nat. Neurosci. 16, 824-831 (2013).

5. Wichmann, F. A. \& Hill, N. J. The psychometric function: I. Fitting, sampling, and goodness of fit. Percept. Psychophys. 63, 1293-1313 (2001). 
6. Pologruto, T. A., Sabatini, B. L. \& Svoboda, K. ScanImage: Flexible software for operating laser scanning microscopes. Biomed. Eng. OnLine 9 (2003).

7. Kim, C. K. et al. Simultaneous fast measurement of circuit dynamics at multiple sites across the mammalian brain. Nat. Methods 13, 325-328 (2016).

8. Xu, N. et al. Nonlinear dendritic integration of sensory and motor input during an active sensing task. Nature 492, 247-251 (2012).

9. Friedrich, J. \& Paninski, L. Fast Active Set Methods for Online Spike Inference from Calcium Imaging. 10.

10. Green, D. M. \& Swets, J. A. Signal Detection Theory and Psychophysics. (John Wiley and Sons, 1966).

11. Felsen, G. \& Mainen, Z. F. Neural Substrates of Sensory-Guided Locomotor Decisions in the Rat Superior Colliculus. Neuron 60, 137-148 (2008).

12. Hand, D. J. \& Till, R. J. A Simple Generalisation of the Area Under the ROC Curve for Multiple Class Classification Problems. Mach. Learn. 45, 171-186 (2001).

13. Dombeck, D. A., Harvey, C. D., Tian, L., Looger, L. L. \& Tank, D. W. Functional imaging of hippocampal place cells at cellular resolution during virtual navigation. Nat. Neurosci. 13, 1433-1440 (2010).

14. Lerner, T. N. et al. Intact-Brain Analyses Reveal Distinct Information Carried by SNc Dopamine Subcircuits. Cell 162, 635-647 (2015).

15. Howard, C. D., Li, H., Geddes, C. E. \& Jin, X. Dynamic Nigrostriatal Dopamine Biases Action Selection. Neuron 93, 1436-1450.e8 (2017).

16. Mulcahy, G., Atwood, B. \& Kuznetsov, A. Basal ganglia role in learning rewarded actions and executing previously learned choices: Healthy and diseased states. PLOS ONE 15, e0228081 (2020).

17. Wei, W. \& Wang, X.-J. Inhibitory control in the cortico-basal ganglia-thalamocortical circuit: complex modulation and its interplay with working memory and decision-making. Neuron 92, 1093-1105 (2016).

18. Wu, Z., Guo, A. \& Fu, X. Generation of low-gamma oscillations in a GABAergic network model of the striatum. Neural Netw. 95, 72-90 (2017). 
a

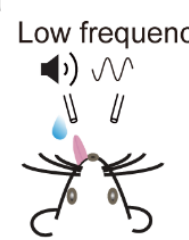

C



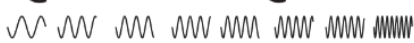

$$
\begin{array}{lllllll}
5 & 6 & 7 & 9 & 11 & 13 & 16
\end{array}
$$



b

Low frequency

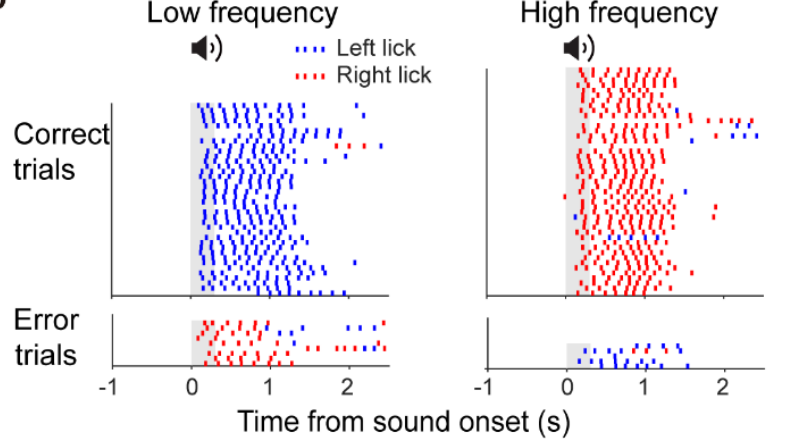

g


d

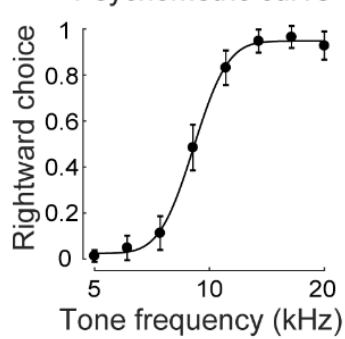

D1-Cre or D2-Cre mice

AAV-hSyn-FLEX-GCaMP6s

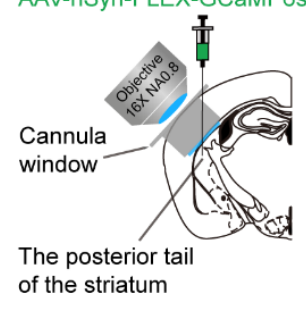

of the striatum

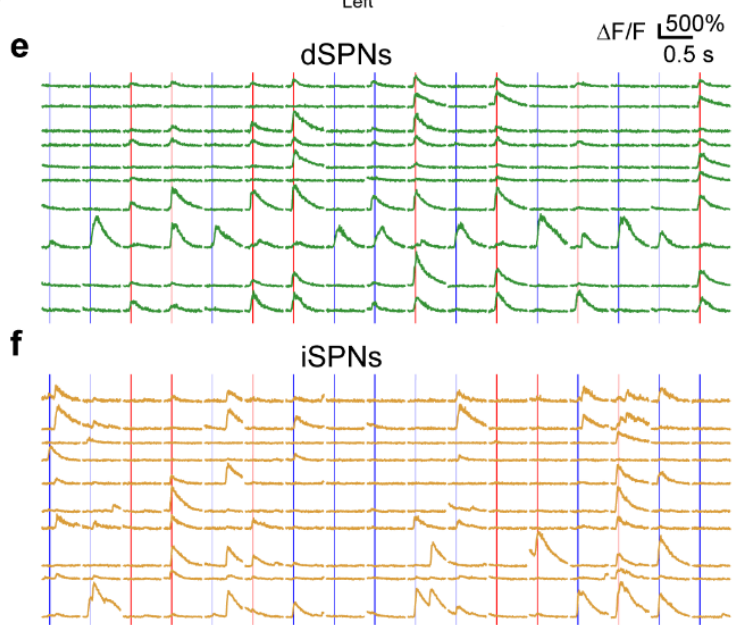

i
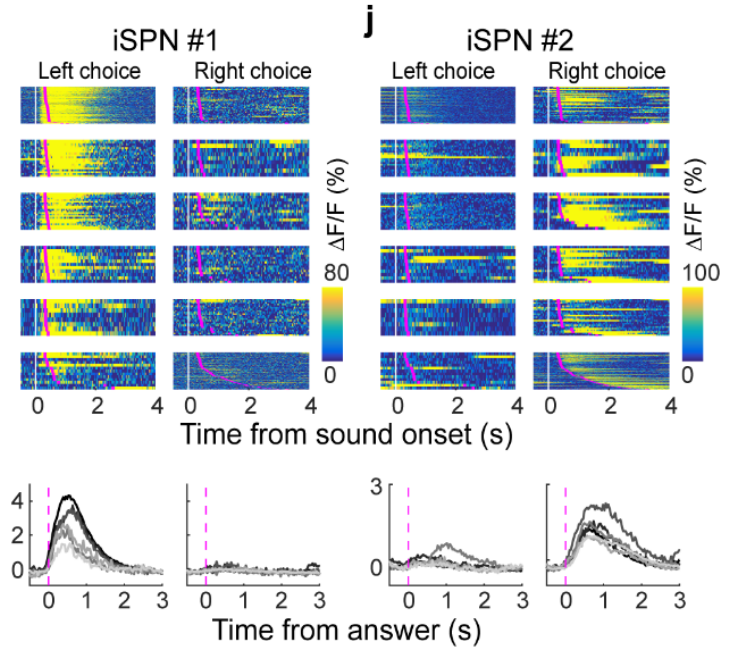

Fig. 1 | Two-photon imaging from dSPNs and iSPNs during sensorimotor decision task.

a, Top, schematic of the behavioral task. Bottom, temporal structure of the task. $\mathbf{b}$, Raster

plot of lick time from an example session (blue, lick right; red, lick left). Grey shadows

indicate the stimulus period. c, Psychometric function from one example behavioral session.

Error bars indicate $95 \%$ confidence interval. d, Schematic showing experimental

configuration of in vivo two-photon imaging from striatal SPNs labeled with GCaMP6s, 
through a chronic cannula window in the TS during behavior (see Methods). e,f, Example traces of calcium signals of dSPNs (e) and iSPNs (f) when mice were performing auditory decision task. The vertical lines indicate mice's answer time (blue, left choice; red, right choice; light blue and light red, error trials). g,h, Color raster plot showing calcium signals $(\Delta \mathrm{F} / \mathrm{F})$ from two example dSPNs with opposite preferences to leftward (g, dSPNs \#1) and rightward (h, dSPNs \#2) choice trials in the same imaging field during a behavioral session. Each row in the color raster represents calcium signals from one trial. Blocks of trials were organized according to tone frequency and choice types. White vertical lines, stimulus onset; magenta lines, answer time. Bottom, mean calcium signals averaged from corresponding blocks of trials aligned to answer times. $\mathbf{i}, \mathbf{j}$, Similar as in $\mathbf{g}$ and $\mathbf{h}$, for two example iSPNs showing opposite preferences to leftward (i, iSPNs \#1) and rightward (j, iSPNs \#2) choices in the same imaging field. 




C
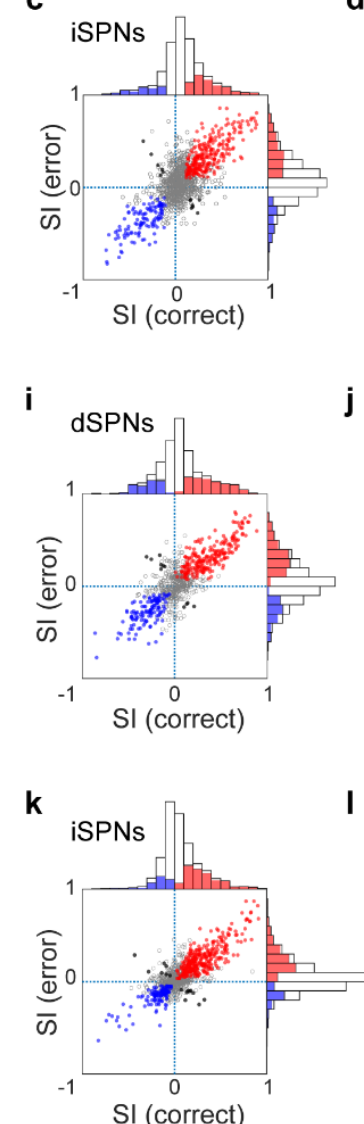

$\mathbf{q}$

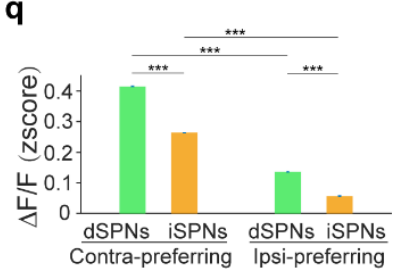

d

I
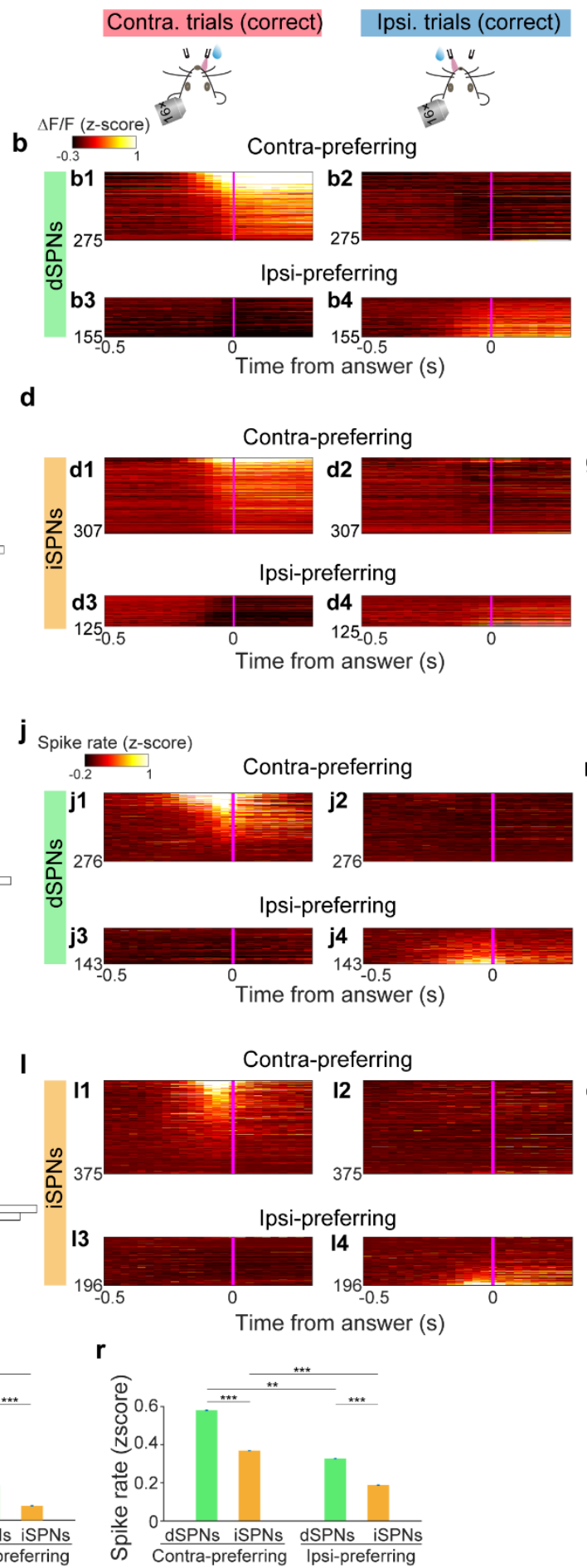

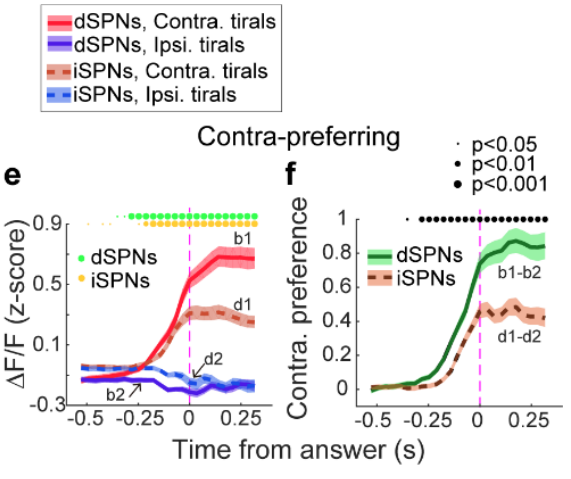

Ipsi-preferring

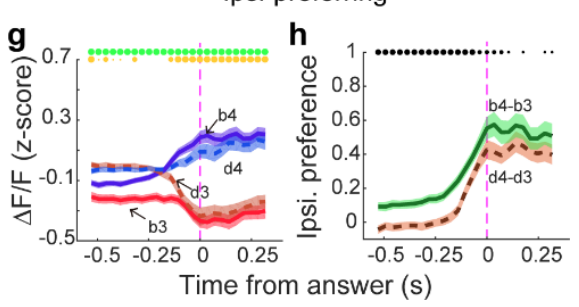

Contra-preferring



Ipsi-preferring

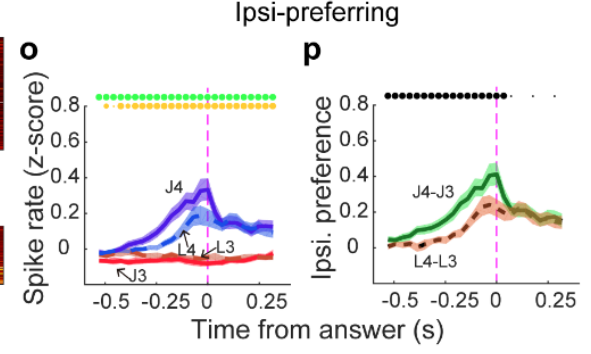

Fig. 2 | dSPNs and iSPNs comprise subpopulations with preference to contralateral or

ipsilateral choices preceding answer time. a, Index of selectivity (SI) for choices calculated using averaged calcium signals in a $100 \mathrm{~ms}$ window before answer time. SIs from correct trials were plotted against SIs from error trials for individual dSPNs. Red, 178/811 neurons with significant contralateral preference; Blue, 142/811 neurons showing significant 
ipsilateral preference; Black, 26/811 neurons with significant sensory category preference; Grey, below significance level, $n=465 / 811$ neurons (see Methods). b, Color raster plots showing z-scored calcium signals of dSPNs with significant selectivity to contralateral (b1, b2) and ipsilateral choices $(\mathbf{b 3}, \mathbf{b 4})$ before answer time. Each row in the color raster represents mean calcium signals from one neuron averaged from correct contralateral trials (left; b1, b3) or ipsilateral trials (right; b2, b4) sorted by SI values. Trials were aligned to answer time (magenta line). c, Similar as in a for iSPNs. Red, contralateral preferring neurons (259/1152); Blue, ipsilateral preferring neurons (117/1152); Black, sensory category selective neurons (13/1152); Grey, below significance level (776/1152). d, Similar as in b for iSPNs. e, Calcium signals averaged across contra-preferring neurons in dSPNs (b1 and b2) and iSPNs (d1 and d2). Shadings indicate bootstrap 95\% confidence interval. Statistical difference level across time between contralateral and ipsilateral trials for each subtype of SPNs are indicated as circles above the plots (paired two-sided Wilcoxon signed rank test). f, Contralateral preference (response difference between contralateral and ipsilateral trials) for contrapreferring dSPNs and iSPNs. Statistical difference level across time between dSPNs and iSPNs are indicated as circles above the plots (two-sided Wilcoxon rank sum test). g, Similar as in e, but for ipsilateral-preferring dSPNs and iSPNs. $\mathbf{h}$, Similar as in $\mathbf{f}$, but for ipsilateral preference (response difference between ipsilateral and contralateral trials) in ipsi-preferring dSPNs and iSPNs. i-p, Similar as in $\mathbf{a - h}$, but using inferred spike rate (z-scored) obtained using calcium signal deconvolution. q, Summary of the calcium signals preceding answer time for all choice selective dSPNs and iSPNs in preferred trial types. $\mathbf{r}$, Similar as in $\mathbf{q}$, but for inferred spike rate (z-scored). Shadings indicate bootstrap 95\% confidence interval. 

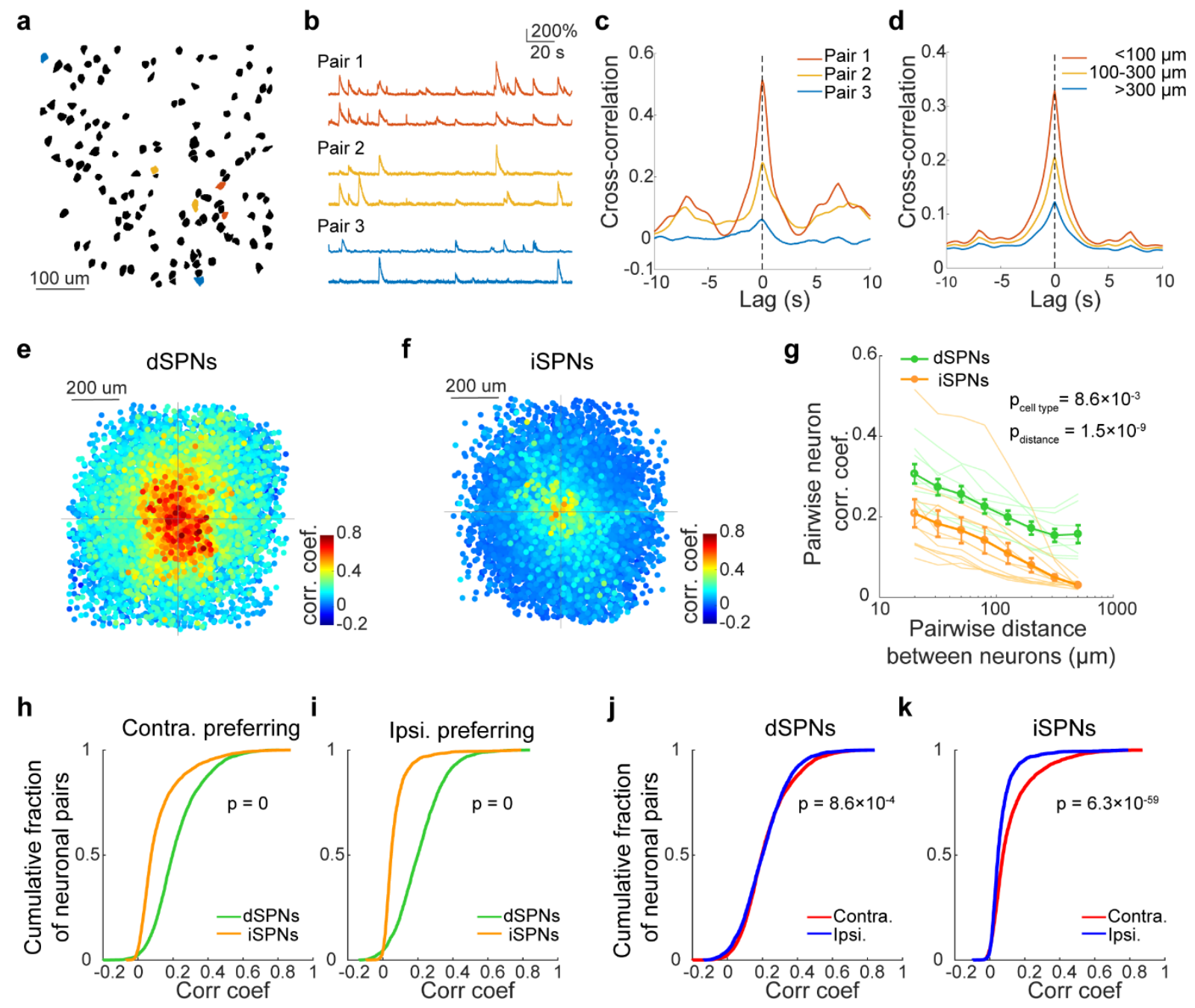

Fig. 3 | Differential spatiotemporal activity patterns of dSPNs and iSPNs.

a, An example field of dSPNs showing the spatial distribution of regions of interest (ROIs).

Colored ROIs indicate pairs of neurons with different spatial distances. Red, $<100 \mu \mathrm{m}$; yellow, 100-300 $\mu \mathrm{m}$; blue, $>300 \mu \mathrm{m}$. b, Calcium traces from the example neuron pairs shown in (a). c, Cross-correlation of calcium signals across time for the neuron pairs shown in (b).

d, Cross-correlation across time averaged for all neuron pairs in the example imaging field as in a with different distances ranges. Shadings indicate SEM. e, Two-dimensional view showing spatiotemporal organization of dSPNs from an example imaging field. The coordinate of each dot represents the relative position between a pair of neurons, with one neuron positioned at the origin. The color indicates the correlation coefficients (CCs) between the calcium traces of the corresponding neuron pairs. The third dimension is ordered 
by the CC values, with higher CC plotted on top layers. f, Similar as in e, for an example imaging field of iSPNs. g. Correlation coefficients between calcium signals of pairs of neurons as a function of the inter-neuronal distance of the corresponding neuron pairs. Each thin line represents data from one imaging field. Green, dSPNs; orange, iSPNs. Data were binned on the dimension of inter-neuronal distance logarithmically scaled for better visualization. Thick lines with error bars, data averaged across imaging fields for dSPNs and iSPNs, respectively. The CCs significantly depend on both the inter-neuronal distance and the cell types, with CCs in dSPNs significantly higher than CCs in iSPNs (Two-way repeatedmeasures ANOVA, Fisher's LSD post hoc tests, $\left.\mathrm{p}_{\text {cell type }}=8.6 \times 10^{-3}, \mathrm{p}_{\text {distance }}=1.5 \times 10^{-9}\right) . \mathbf{h}$, Cumulative distribution functions (CDF) of CCs from contralateral-preferring dSPNs and iSPNs ( $p=0$, two-sample Kolmogorov-Smirnov test, one-tailed). i, CDF of CCs from ipsilateral-preferring dSPNs and iSPNs ( $\mathrm{p}=0$, two-sample Kolmogorov-Smirnov test, onetailed). $\mathbf{j}$, CDF of CCs from contralateral- and ipsilateral-preferring dSPNs ( $p=8.6 \times 10^{-4}$, two-sample Kolmogorov-Smirnov test, one-tailed). $\mathbf{k}$, CDF of CCs from contralateral- and ipsilateral-preferring iSPNs $\left(\mathrm{p}=6.3 \times 10^{-59}\right.$, two-sample Kolmogorov-Smirnov test, onetailed). 



\section{Fig. 4 | Axonal activity of dSPNs in SNr shows dominant preference for contralateral}

choices. a, Experimental configuration of fiber photometry recording of axonal activity of dSPNs in SNr. Top, schematic showing fiber photometry recording system. Bottom, schematic showing virus injection sites in the TS and the fiber implantation in the SNr. b, Calcium signals from dSPN axons in $\mathrm{SNr}$ from an example behavioral session showing greater responses in contralateral trials than in ipsilateral trials. Each row in the color raster plot represents calcium signals from one trial. The calcium signal trials were sorted according to trial types as indicated on the left. White vertical lines, stimulus onset; magenta lines, answer time. Bottom, mean calcium signal traces averaged for the four trial types, aligned to answer times. Shadings indicate SEM. c, Comparison of axonal activity between contralateral and ipsilateral correct trials. Each dot represents one recording session $(n=12$ sessions from 7 mice, $\mathrm{p}<0.05$, paired two-sided Wilcoxon signed rank test; see Methods). d, Selectivity index of dSPN axon activity in $\mathrm{SNr}$ for contralateral and ipsilateral trials calculated from correct trials plotted against selectivity index calculated from error trials. Each dot 
bioRxiv preprint doi: https://doi.org/10.1101/2021.11.16.468594; this version posted November $19,2021$. The copyright holder for this

preprint (which was not certified by peer review) is the author/funder, who has granted bioRxiv a license to display the preprint in perpetuity. It is made available under aCC-BY-NC-ND 4.0 International license.

corresponds to one recording session. Magenta dots indicate sessions with significant SI for contra- and ipsilateral choices in correct trials (see Methods). 
a

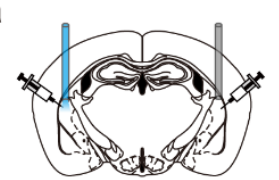

D1-Cre mice

AAV-CAG-FLEX-ChR2

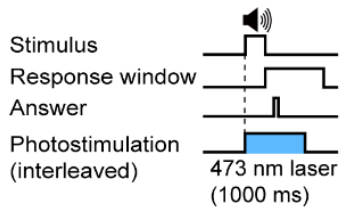

e

iSPNs activation

D2-Cre mice

AAV-CAG-FLEX-ChR2

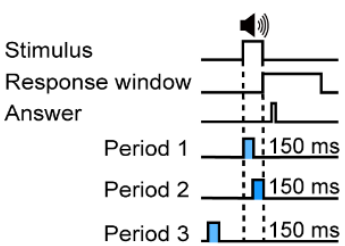

Period 3 几 : $150 \mathrm{~ms}$

i



D1-Cre mice

AAV-Ef1a-DIO-eNpHR3.0

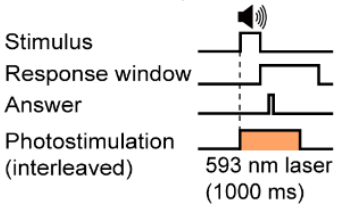

Photostimulation (1000 ms) b



f

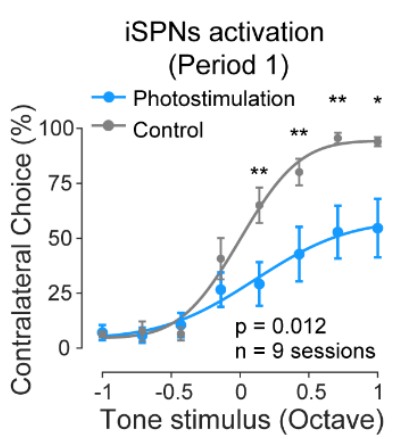

j



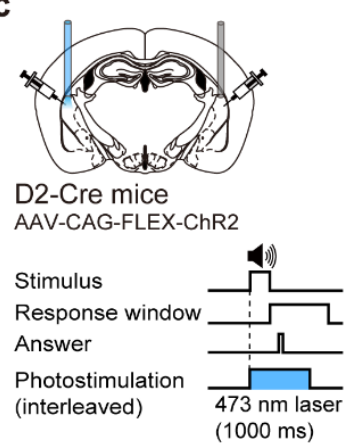

g

iSPNs activation (Period 2)

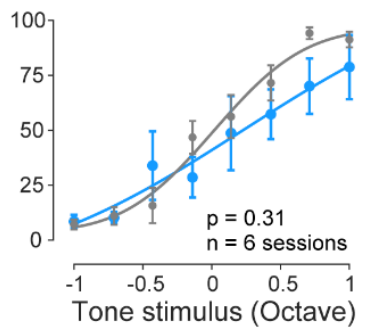

k



m

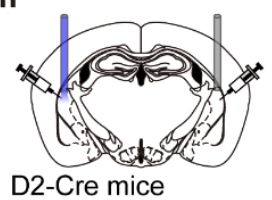

AAV-hSyn-SIO-stGtACR2

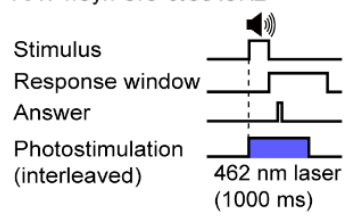

d

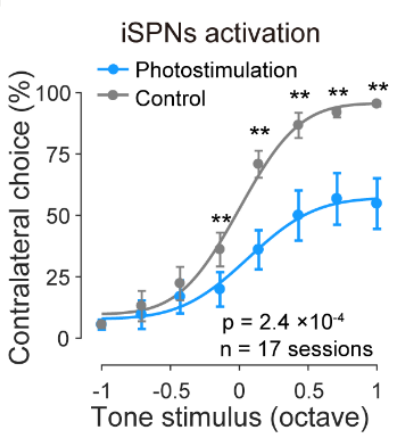

h

iSPNs activation

(Period 3)
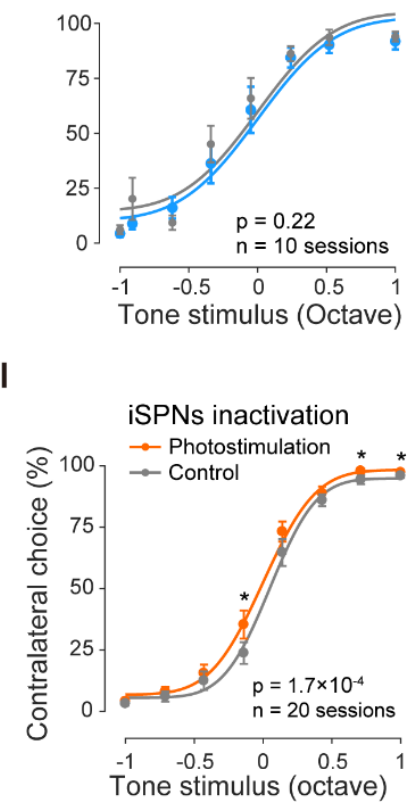

n

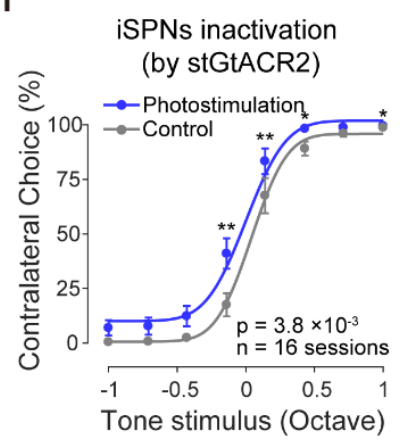

Fig. 5 | Unilateral optogenetic perturbation of dSPNs and iSPNs bidirectionally

regulates competing choices. a, Schematic showing experimental configuration for

optogenetic activation of dSPNs (see Methods). b, Summarized psychometric functions from control trials (grey) and trials with dSPN activation (blue). Percentage of contralateral choice (relative to photostimulated hemisphere) is plotted against tone stimuli in relative octaves 
(positive values associated with contralateral choices; negative values associated with ipsilateral choices). $\mathrm{n}=22$ sessions from $11 \mathrm{D} 1$-Cre mice; $\mathrm{p}=8.9 \times 10^{-3}$ for photostimulation effect, two-way repeated-measures ANOVA; ${ }^{*} \mathrm{p}<0.01$, Fisher's LSD post hoc tests. Miss (no lick response) trials were not included. c, Schematic showing unilateral optogenetic activation of iSPNs, similar as in a. d, Summarized psychometric functions from control trials and trials with iSPN activation, similar as in $\mathbf{b} . \mathrm{n}=17$ sessions from 9 D2-Cre mice; $\mathrm{p}=$ $2.4 \times 10^{-4}$ for photostimulation effect, two-way repeated-measures ANOVA; **p $<0.01$, Fisher's LSD post hoc tests. e, Schematic showing optogenetic activation of iSPNs at different periods. Blue shades indicate the period of photostimulation. Period 1, a $150 \mathrm{~ms}$ window following sound onset; Period 2, a $150 \mathrm{~ms}$ window before the end of tone stimulus; Period 3, a $150 \mathrm{~ms}$ window starting from $650 \mathrm{~ms}$ before sound onset. f, Summarized psychometric functions similar as in $\mathbf{d}$ for iSPN activation in period 1. $\mathrm{n}=9$ sessions from 6 D2-Cre mice; $p=0.012$ for photostimulation effect, two-way repeated-measures ANOVA; * $p$ $<0.05,{ }^{*} \mathrm{p}<0.01$, Fisher's LSD post hoc tests. $\mathbf{g}$, Similar as in $\mathbf{f}$ for iSPN activation in period 2. $\mathrm{n}=6$ sessions from 3 D2-Cre mice; $\mathrm{p}=0.31$ for photostimulation effect, two-way repeated-measures ANOVA. $\mathbf{h}$, Similar as in $\mathbf{f}$ for iSPN activation in period 3. $\mathrm{n}=10$ sessions from 3 D2-Cre mice; $p=0.22$ for photostimulation effect, two-way repeated-measures ANOVA. i, Schematic showing experimental configuration for unilateral optogenetic inactivation of dSPNs using eNpHR3.0. $\mathbf{j}$, Summarized psychometric functions as in $\mathbf{b}$ for unilateral dSPN inactivation. $\mathrm{n}=12$ sessions from 6 D1-Cre mice; $\mathrm{p}=3.8 \times 10^{-3}$ for photostimulation effect, two-way repeated-measures ANOVA; ${ }^{*} p<0.05 ;{ }^{* *} p<0.01$, Fisher's LSD post hoc tests. k, Schematic for unilateral iSPN inactivation using eNpHR3.0. l, Summarized psychometric functions as in $\mathbf{j}$ for unilateral iSPN inactivation. $\mathrm{n}=20$ sessions from 10 D2-Cre mice; $p=1.7 \times 10^{-4}$ for photostimulation effect, two-way repeated-measures ANOVA; ${ }^{*} \mathrm{p}<0.05$, Fisher's LSD post hoc tests. m, Schematic for unilateral iSPN 
inactivation using stGtACR2. n, Summarized psychometric functions for unilateral iSPN inactivation using stGtACR2, similar as in $\mathbf{l} . \mathrm{n}=16$ sessions from 8 D2-Cre mice; $\mathrm{p}=$ $3.8 \times 10^{-3}$ for photostimulation effect, two-way repeated-measures ANOVA; ${ }^{*} \mathrm{p}<0.05, * * \mathrm{p}<$ 0.01, Fisher's LSD post hoc tests. 
a

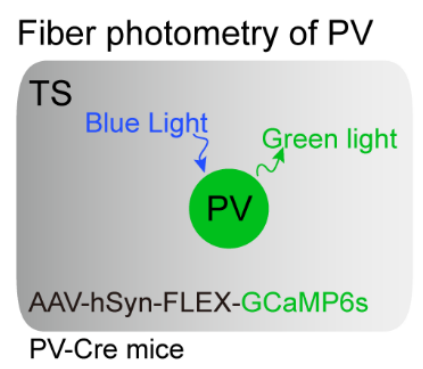

C



b
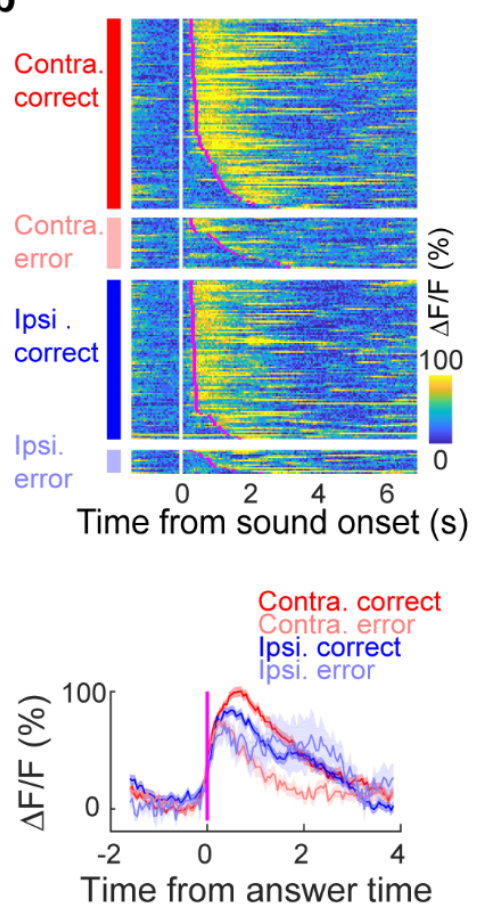

d

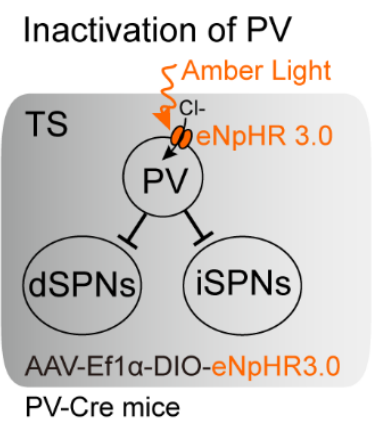

Choice behavior

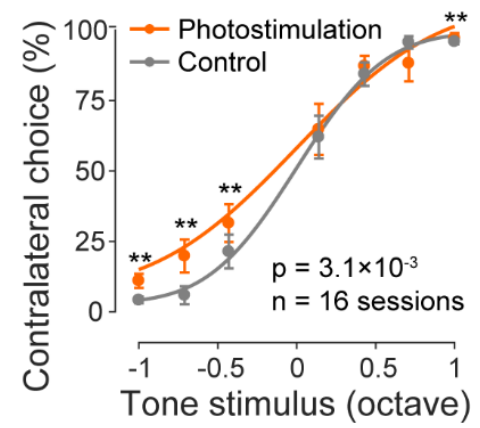

Fig. 6 | Concurrent disinhibition of dSPNs and iSPNs biases choices towards the

contralateral side. a, Schematic showing fiber photometry recording from PV interneurons

in TS. $\mathbf{b}$, Calcium signals of PV interneurons in the TS from an example behavior session.

Upper, color raster plot of calcium signals from individual trials sorted according to the four trial types; lower, mean traces averaged for different trial types (Similar as in Fig. 4b). c, Summarized responses of PV interneurons compared between trials with contralateral and ipsilateral choices. $\mathrm{n}=12$ sessions from 6 mice, $\mathrm{p}=0.27$, paired two-sided Wilcoxon signed rank test. d, Schematic showing optogenetic inactivation of PV interneuron in TS. e, Summarized psychometric functions following unilateral PV interneuron inactivation, similar as in Fig. 6j. $\mathrm{n}=16$ sessions from 8 PV-Cre mice; $\mathrm{p}=3.1 \times 10^{-3}$ for photostimulation effect, two-way repeated-measures ANOVA; **p $<0.01$, Fisher's LSD post hoc tests. 
a

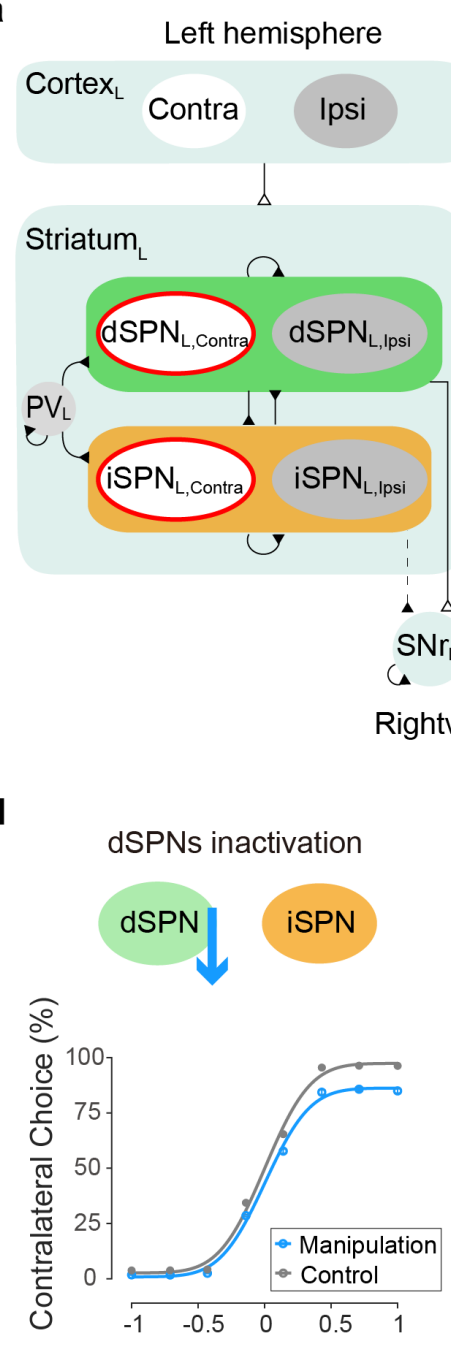

g



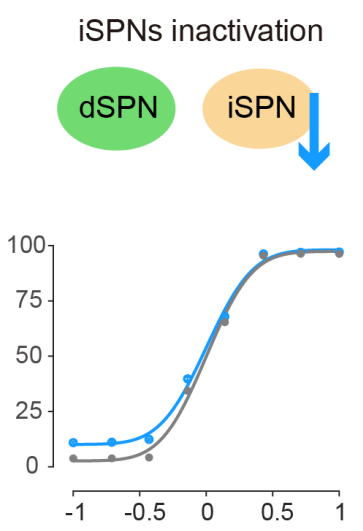

h

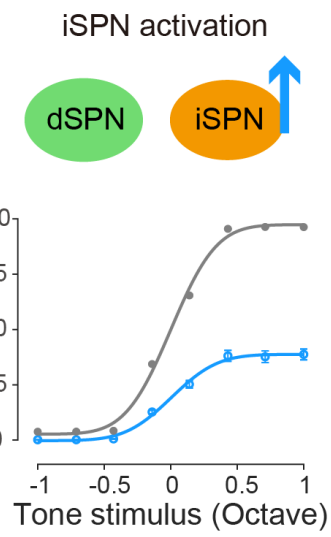

b

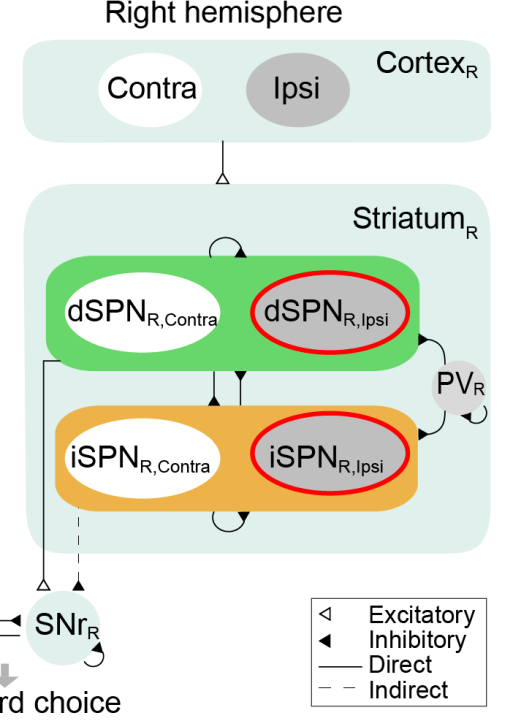



C

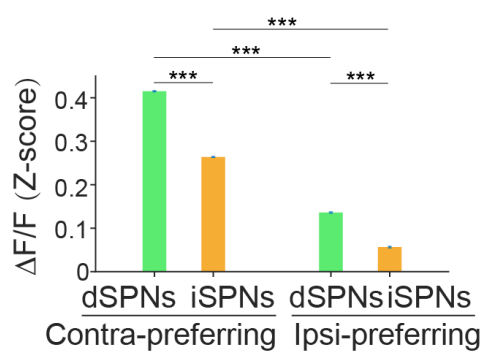

f

PV inactivation

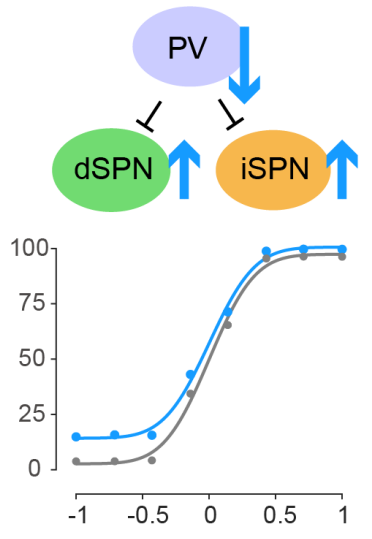

i

Symmetrical dSPN and iSPN



Fig. $7 \mid$ A neural network model recapitulates the causal roles of the dSPNs and iSPNs in

perceptual decision behavior. a, Schematic showing the network model organization. L, left hemisphere; R, right hemisphere; Contra, contralateral preferring neuronal population; Ipsi, ipsilateral preferring neuronal population. See Methods. b, Responses of modeled dSPNs and 
iSPNs with contralateral or ipsilateral preference in preferred trial types. ${ }^{* * *}, \mathrm{p}<0.001$, twosided Wilcoxon rank sum test. c, Experimental data of dSPN and iSPN responses same as in Fig. 2q. d-h, Model simulation of behavioral effects of optogenetic manipulations. Model outputs of choice behavior from control trials were shown in grey, and those from trials with unilateral dSPN activation (d), iSPN activation (e), dSPN inactivation (f), iSPN inactivation (g) and PV interneuron inactivation (h) were shown in blue. Each curve was obtained from 22 different $I^{\text {stim }}$ values (Table S2). i, Simulated choice behavior when dSPN and iSPN activity are symmetrical. 


\section{Extended Data}

\section{Asymmetrical choice-related ensemble activity in direct and indirect- pathway striatal neurons drives perceptual decisions}

Lele Cui ${ }^{1,2,6}$, Shunhang Tang ${ }^{1,2,6}$, Kai Zhao ${ }^{4}$, Jingwei Pan ${ }^{1}$, Zhaoran Zhang ${ }^{5}$, Bailu $\mathrm{Si}^{4}$, Ninglong $\mathrm{Xu}^{1,2,3,7 *}$

${ }^{1}$ Institute of Neuroscience, State Key Laboratory of Neuroscience, CAS Center for Excellence in Brain Science and Intelligence Technology, Chinese Academy of Sciences, Shanghai 200031, China

${ }^{2}$ School of Future Technology, University of Chinese Academy of Sciences, Beijing 100049, China

${ }^{3}$ Shanghai Center for Brain Science and Brain-Inspired Intelligence Technology, Shanghai 201210, China

${ }^{4}$ School of Systems Science, Beijing Normal University, Beijing, 100875, China

${ }^{5}$ School of Life Sciences, Sichuan University, Chengdu, Sichuan 610064, China

${ }^{6}$ These authors contributed equally

*Correspondence: xunl@ion.ac.cn 
a

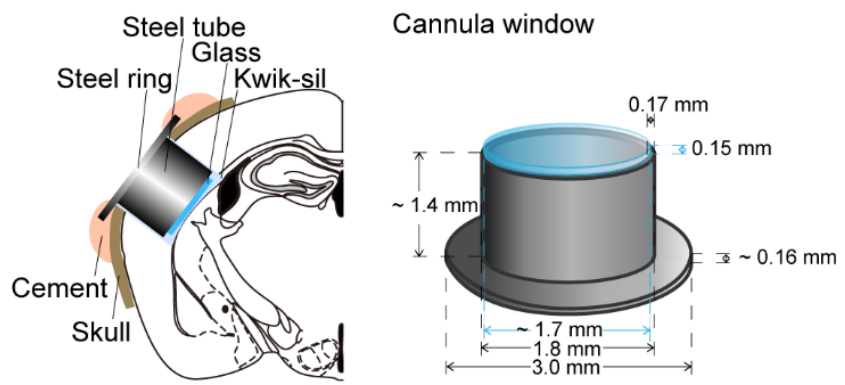

b

D1-Cre mouse injected with AAV-FLEX-GFP (direct pathway)
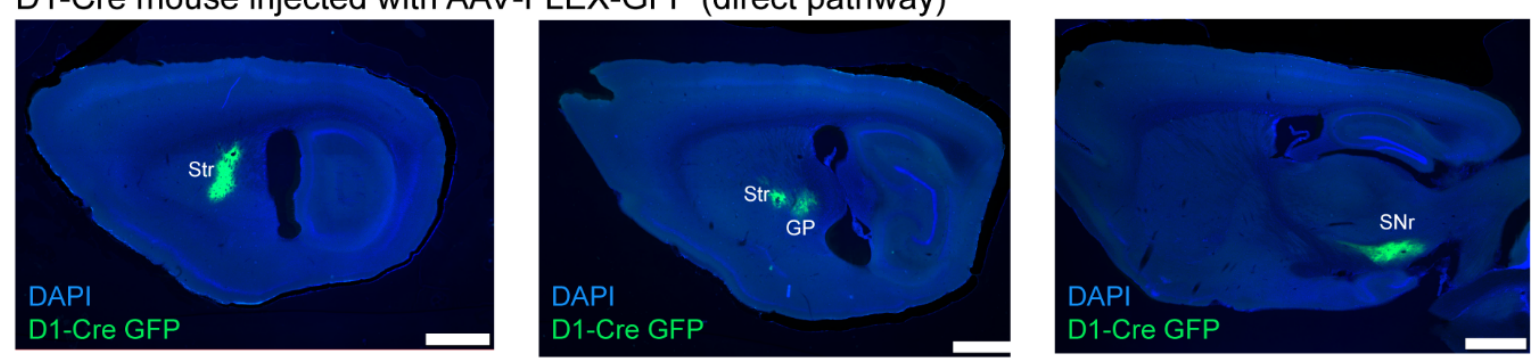

C

D2-Cre mouse injected with AAV-FLEX-GFP (indirect pathway)
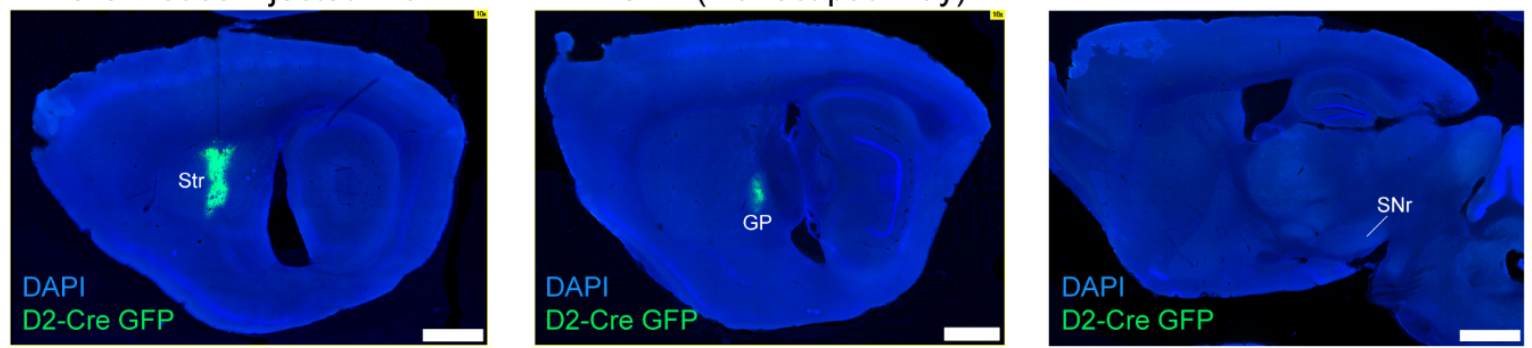

Extended Data Fig. 1 | Cannular window for chronic in vivo two-photon calcium imaging from the posterior tail of the striatum (TS) and histology verifying the cell type specific virus labeling of SPNs. a, Schematic showing chronically implanted cannular window for in vivo two-photon imaging of the TS. b, Sagittal section from an example D1Cre mouse, showing Cre-dependent viral expression (GFP) in the TS and downstream axonal targets (GP and SNr) of labeled dSPNs. c, Sagittal section from an example D2-Cre mouse, showing Cre-dependent viral expression (GFP) in the TS and downstream axonal targets (GP only) of labeled neurons. Scale bars, $1 \mathrm{~mm}$. 
a
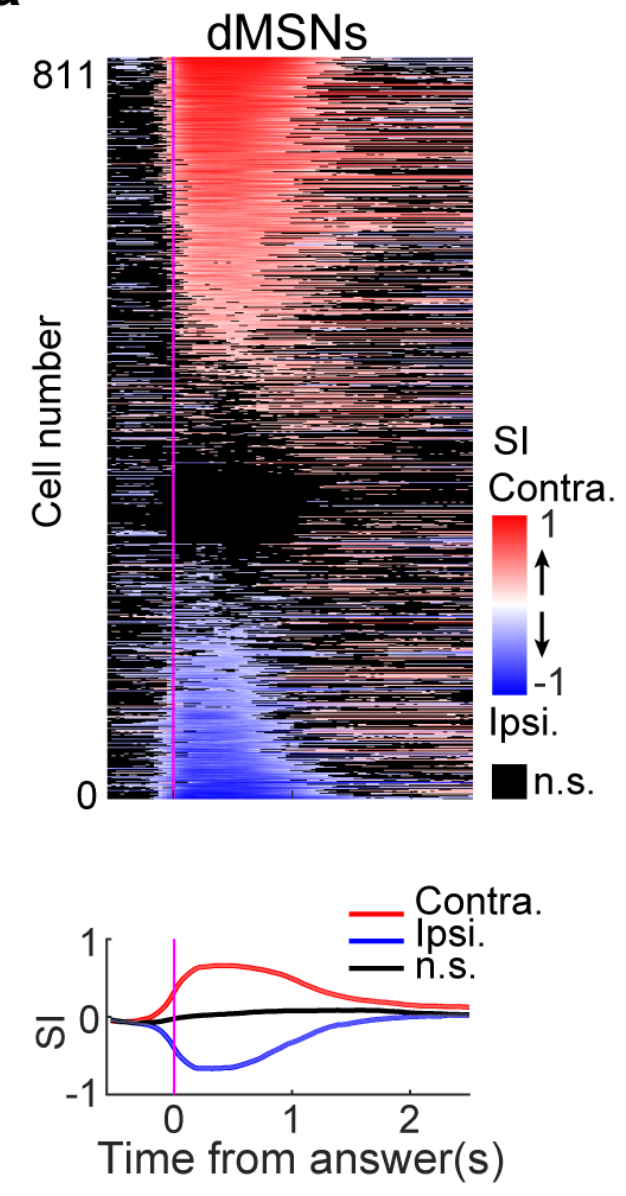

b
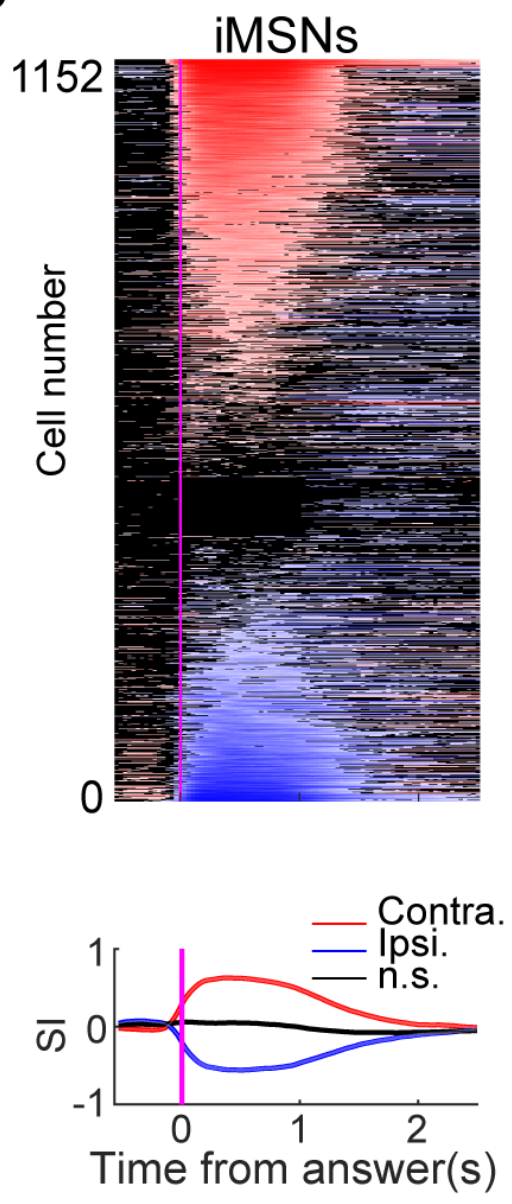

\section{Extended Data Fig. 2 | Differential population selectivity within and between dSPNs and}

iSPNs. a, Selectivity index (SI) for contra- and ipsilateral choices across the time course of trials for all recorded dSPNs $(n=811$ neurons from 8 imaging fields of 4 mice). 'Contra.' and 'Ipsi' refer to the direction of choice relative to the imaged hemisphere. Each row represents the selectivity index from a single neuron. Neurons are sorted by their mean selectivity index within $1 \mathrm{~s}$ after mice's answer time. Magenta line, answer time. Bottom, traces of mean selectivity index averaged for the three types of neurons (significantly selective to contralateral or ipsilateral choices, or no significant selectivity), aligned to answer times. see Methods). Shadings indicate SEM. b, SI averaged within $1 \mathrm{~s}$ after mice's answer time, calculated in correct trials plotted against SI calculated during error trials for all recorded dSPNs. Each data point corresponds to one neuron. Red, showing significant preference for contralateral choices; Blue, showing significant preference for ipsilateral choices; black, 
bioRxiv preprint doi: https://doi.orq/10.1101/2021.11.16.468594: this version posted November 19.2021 . The copyriaht holder for this

preprint (which was not certified by peer review) is the author/funder, who has granted bioRxiv a license to display the preprint in perpetuity. It is made available under aCC-BY-NC-ND 4.0 International license.

significant sensory category preference; grey, below significance level (see Methods).

Shadings indicate SEM. 

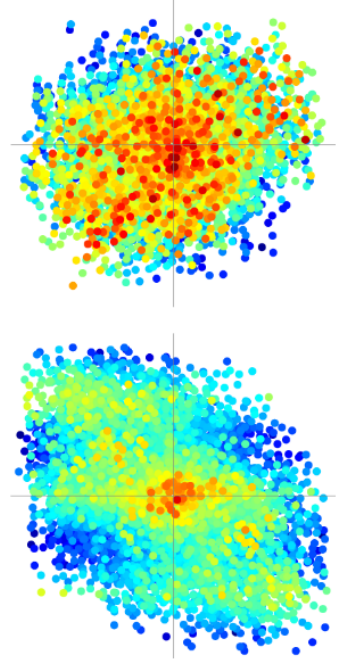

b


dSPNs


iSPNs


\section{Extended Data Fig. 3 | Spatiotemporal correlation of dSPNs and iSPNs for individual}

imaging fields. a, Spatiotemporal correlation of dSPNs from 8 imaging fields of 4 D1-Cre mice, similar as in Fig. 3e. b, Spatiotemporal correlation of iSPNs from 11 imaging fields of 6 D2-Cre mice, similar as in Fig. 3f. 
a Optogenetic activation (cell-type specific)

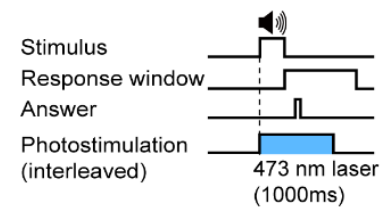

d

Optogenetic inactivation by NpHR3

(cell-type specific)

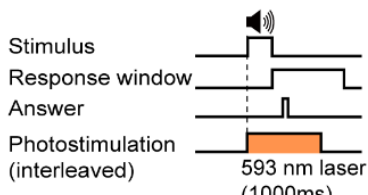

h

Optogenetic inactivation by stGtACR2 (cell-type specific)

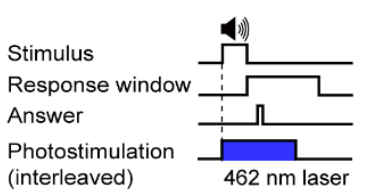

b

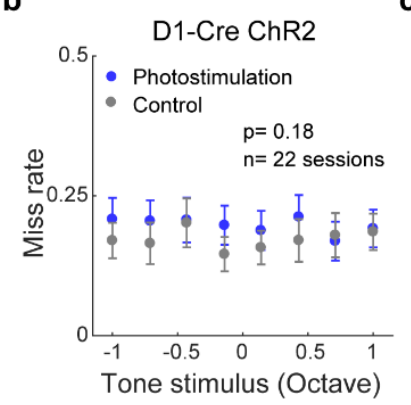

e



i

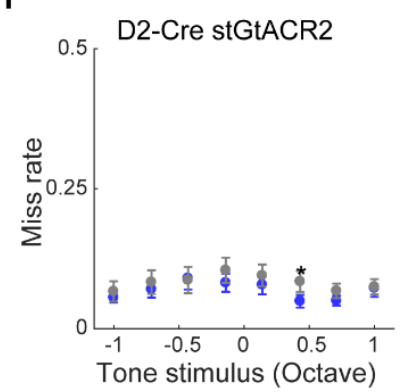

C

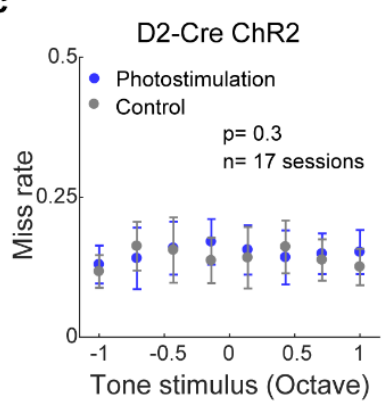

f



g

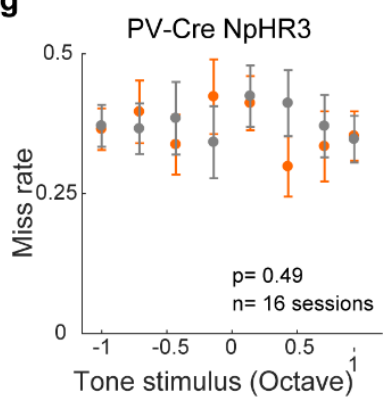

Extended Data Fig. 4 | Effects of optogenetic manipulations on miss rate. a, Schematic

showing trial structure with optogenetic activation. $\mathbf{b}$, Miss rate as a function of tone stimulus in trials with or without optogenetic activation of dSPNs. $\mathbf{c}$, As in $\mathbf{b}$ for optogenetic activation of iSPNs. d, Schematic showing trial structure with optogenetic inactivation using eNpHR3.0. e, Similar as in $\mathbf{b}$ for optogenetic inactivation of dSPNs. $\mathbf{f}$, Similar as in $\mathbf{b}$ for optogenetic inactivation of iSPNs. g, Similar as in $\mathbf{b}$, for optogenetic inactivation of PV interneurons. $\mathbf{h}$, Schematic showing trial structure with optogenetic inactivation of iSPNs using stGtACR2. i, Similar as in $\mathbf{f}$ for optogenetic inactivation of iSPNs using stGtACR2. P values shown are for effects of photostimulation from two-way repeated-measures ANOVA. 
a

dSPN activation

D1-Cre mice

AAV-CAG-FLEX-ChR2

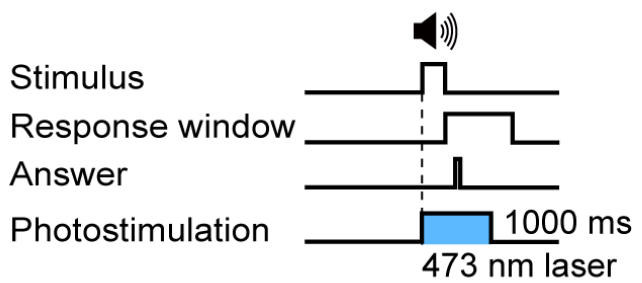

C

iSPN activation

D2-Cre mice

AAV-CAG-FLEX-ChR2



b

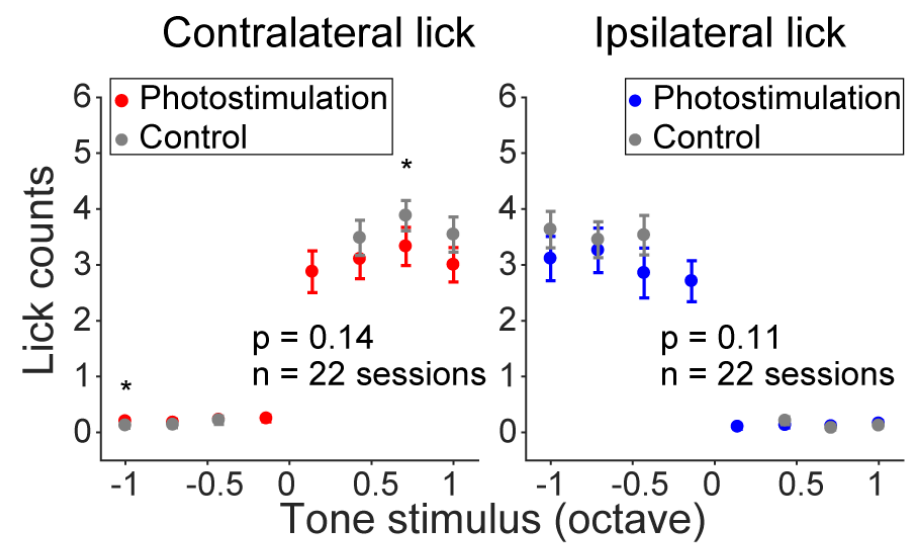

d

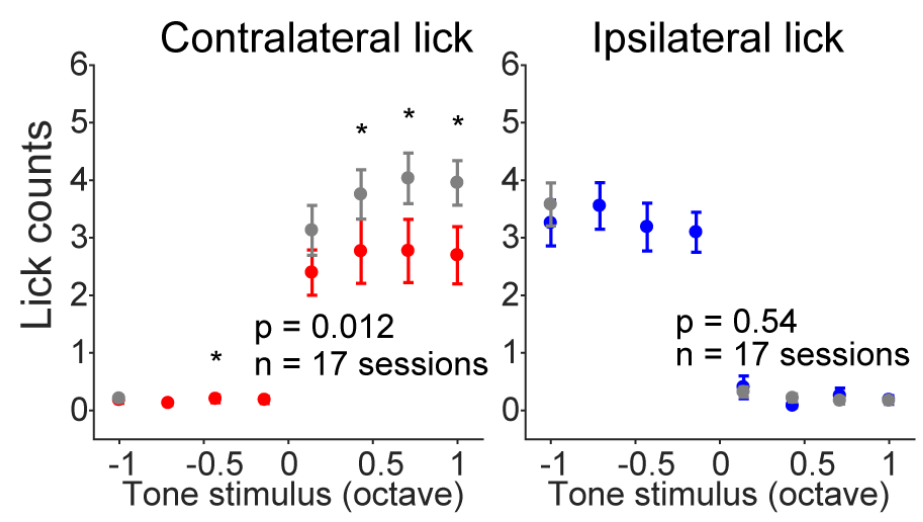

Extended Data Fig. 5 | The effects on lick rate of optogenetic activation. a, Schematic showing trial structure with optogenetic activation of dSPNs. b, Number of licks within the period of photostimulation as a function of tone stimulus from trials with or without photostimulation. Only correct trials were included. Left, contralateral licking. Right, ipsilateral licking. c, Schematic showing trial structure with optogenetic activation of iSPNs. d, Similar as in $\mathbf{b}$ for optogenetic activation of iSPNs. P values shown are for effects of photostimulation from two-way repeated-measures ANOVA. 


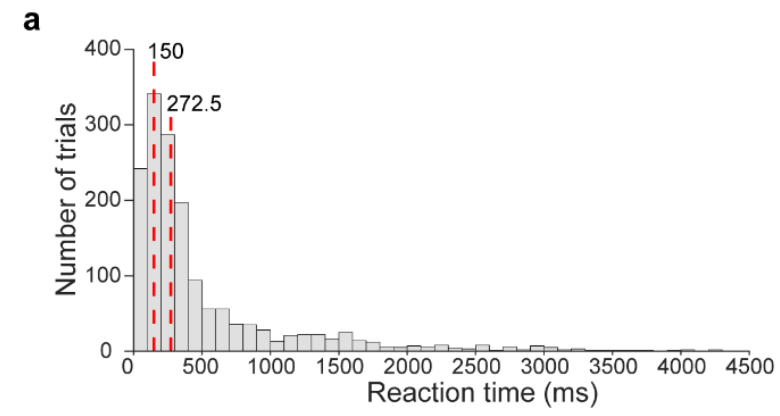

b

Optogenetic activation (cell-type specific)

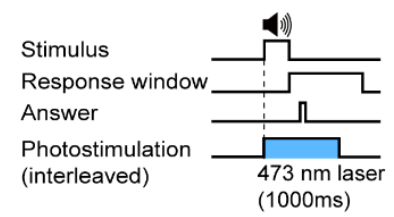

\section{e}

Optogenetic inactivation by NpHR3 (cell-type specific)

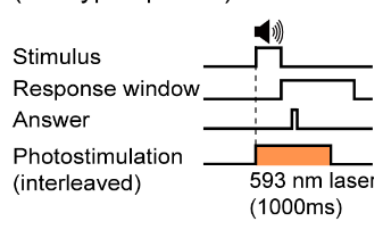

i

Optogenetic inactivation by stGtACR2 (cell-type specific)

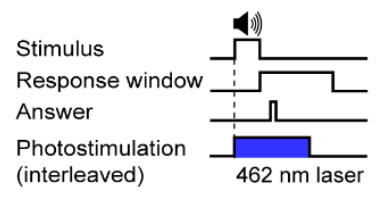

C



f

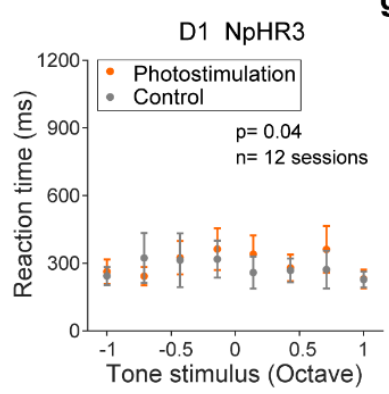

j

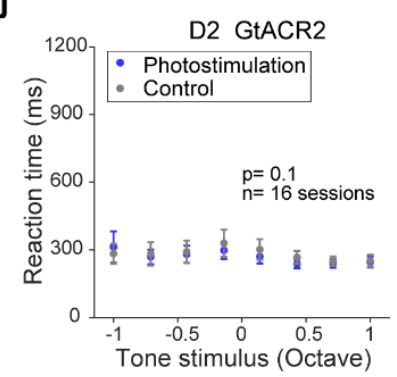

d

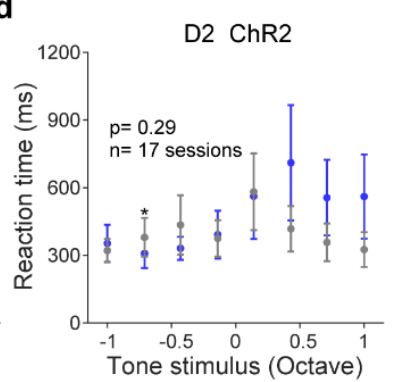

g


\section{Extended Data Fig. 6 | The effects of optogenetic manipulation on mice's reaction time.}

a, Distribution of reaction time in control trials for D2-Cre mice as shown in Fig. 5d. Vertical red lines indicate the first quarter $(150 \mathrm{~ms})$ and the median $(272.5 \mathrm{~ms}) . \mathbf{b}$, Schematic showing trial structure with optogenetic activation. c, Reaction time as a function of tone stimulus in trials with or without optogenetic activation of dSPNs. $\mathbf{d}$, As in $\mathbf{c}$ for iSPN activation. e, Schematic showing trial structure with optogenetic inactivation with eNpHR3.0. f-h Similar as in $\mathbf{c}$ for dSPN inactivation (f), iSPN inactivation (g), and PV interneuron inactivation (h). i, 
Schematic showing trial structure with optogenetic inactivation with stGtACR2. j, Similar as in $\mathbf{c}$ for iSPN inactivation with stGtACR2.

$P$ values shown are for effects of photostimulation from two-way repeated-measures ANOVA. $* \mathrm{p}<0.05$, Fisher's LSD post hoc tests. 
a

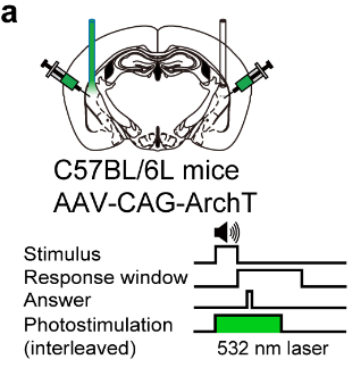

d

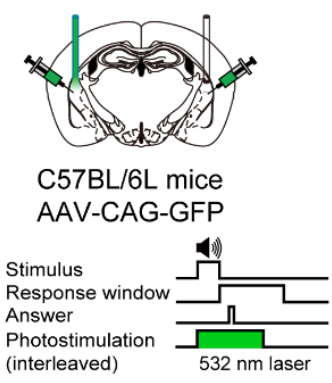

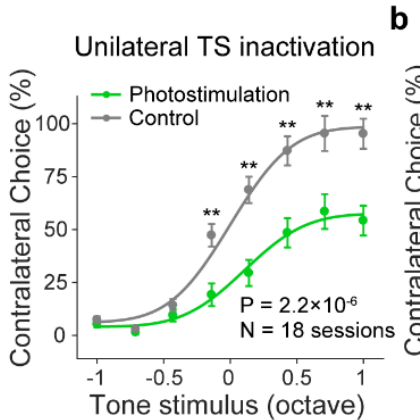

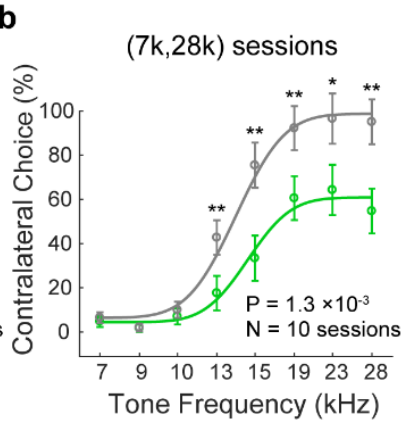

e

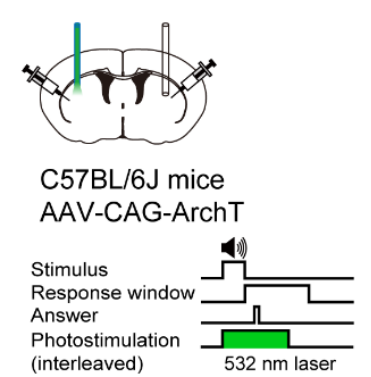

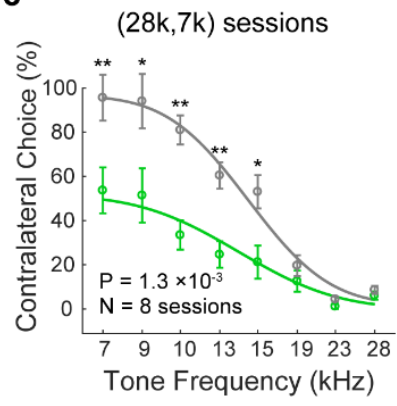

Inactivation of unilateral dorsal striatum



\section{Extended Data Fig. 7 | Unilateral optogenetic inactivation of the TS and the anterior}

sub-region of the dorsal striatum. a, AAV-CAG-ArchT was used to inactivate the TS during the auditory decision task. Summarized psychometric functions from all sessions $(n=18$ sessions from 9 mice). b-c, Psychometric functions from two groups of mice trained with reversed stimulus-choice association resepctively. $\mathrm{n}=10$ sessions from 5 mice in $\mathbf{b} . \mathrm{n}=8$ sessions from 4 mice in c. $\mathbf{d}$, Similar as in $\mathbf{a}$, in control group injected with AAV-CAG-GFP in the TS, $n=12$ sessions from 3 mice. e, Similar as in a for optogenetic inactivation of the anterior sub-region of the dorsal striatum, injected with AAV-CAG-ArchT, $\mathrm{n}=11$ sessions from 6 mice. P values shown are for effects of photostimulation from two-way repeatedmeasures ANOVA. $* \mathrm{p}<0.05, * * \mathrm{p}<0.01$, Fisher's LSD post hoc tests. 
a

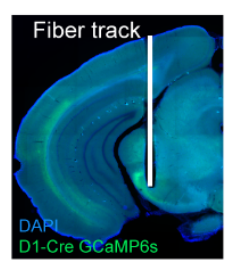

AAV-hSyn-FLEXGCaMP6s

C

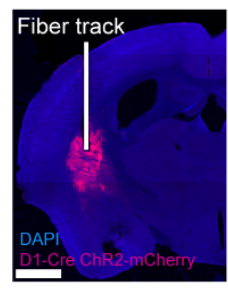

AAV-CAG-FLEXChR2-mCherry

d

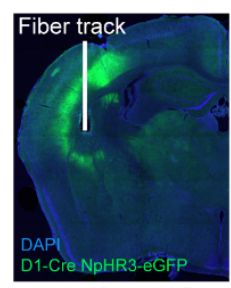

AAV-Ef1a-DIOeNpHR3.0-eYFP

e

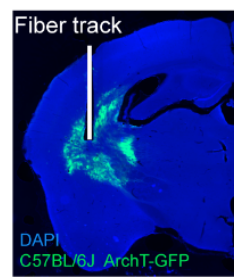

AAV-CAG-ArchTGFP

f

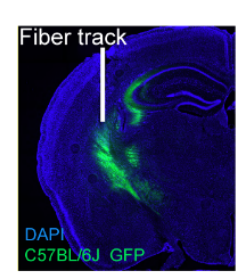

AAV-CAG-GFP
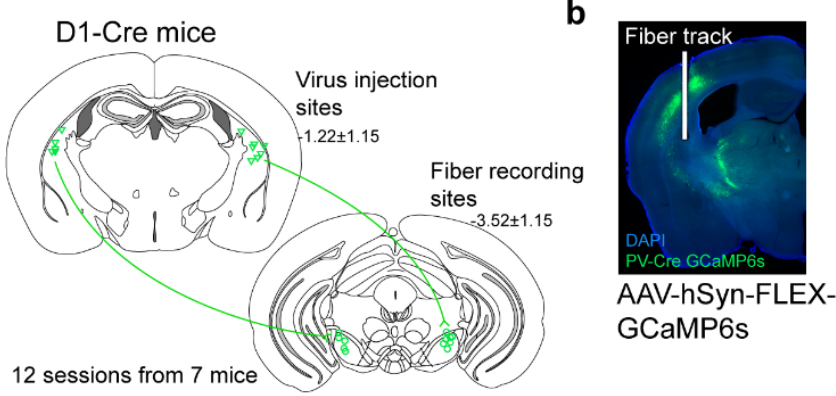

GCaMP6s

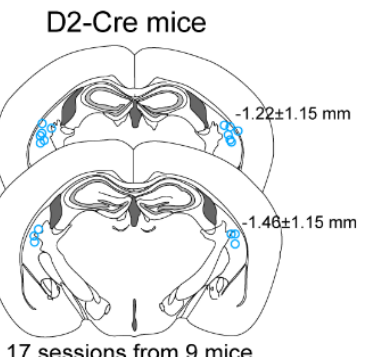

22 sessions from 11 mice

17 sessions from 9 mice

\section{D1-Cre mice}

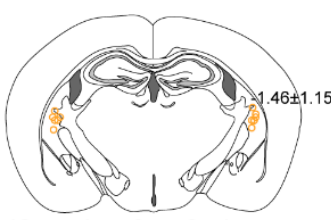

12 sessions from 6 mice

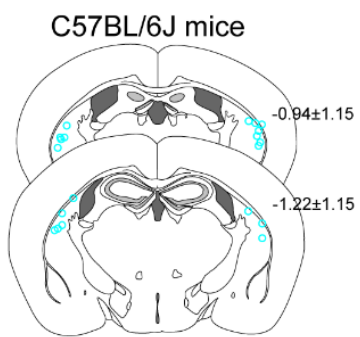

12 sessions from 6 mice

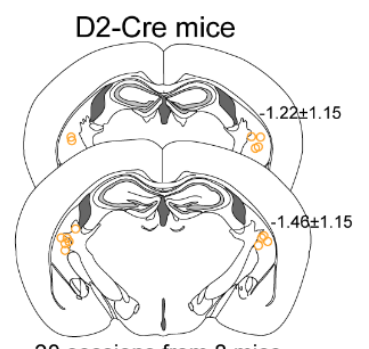

20 sessions from 8 mice

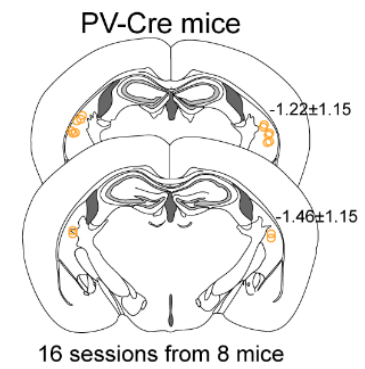

C57BL/6J mice

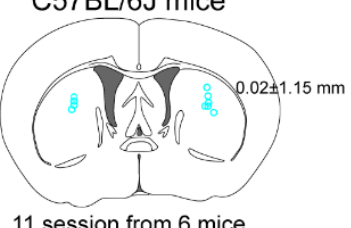

11 session from 6 mice



12 sessions from 6 mice

\section{Extended Data Fig. 8 | Histological images of virus injection and fiber track for fiber}

photometry and optogenetic manipulations. a, Left, coronal histological sections from one representative D1-Cre mouse injected with AAV-FLEX-GCaMP6s in the TS and implanted with fiber optics in SNr. Right, schematic illustrating the virus injection sites (green triangles) and the fiber recording sites (green circles) in all experiments. b, Left, example histological 
image of a PV-Cre mouse injected with AAV-FLEX-GCaMP6s in the TS. Right, locations of fiber tips (green circles) in all experiments. c, Left, example histological image of a D1-Cre mouse injected with AAV-FLEX-ChR2-mCherry in the TS. Right, locations of fiber tips (blue circles) for optogenetic activation in all D1-Cre and D2-Cre mice, respectively. d, Left, example histological image of a D1-Cre mouse injected with AAV-DIO-eNpHR3.0-eYFP in the TS. Right, the locations of fiber tips (orange circles) for optogenetic inactivation in D1Cre, D2-Cre and PV-Cre mice in all experiments. e, Left, example histological image of a C57BL/6J mouse injected with AAV-CAG-ArchT-GFP in the TS. Right, the locations of fiber tips (cyan circles) for optogenetic inactivation of the TS and the anterior sub-region of the dorsal striatum in all experiments. f, Left, example histological image of a C57BL/6 J mouse injected with AAV-CAG-GFP in the TS. Right, locations of fiber tips (cyan circles) in all experiments. 
Extended Data Tab. 1 | Parameters for the network model.

\begin{tabular}{|c|c|c|c|c|c|c|c|}
\hline Parameter & Value & Parameter & Value & Parameter & Value & Parameter & Value \\
\hline $\begin{array}{c}C T X_{R, I p s i} \\
w_{D_{1}, R, \text { Contra }}^{C}\end{array}$ & 0.04 & $w_{D_{1, R, \text { Contra }}}^{C T X_{R, \text { Contra }}}$ & 0.80 & $w_{P V_{L}}^{C T X_{L}}$ & 0.015 & $w_{S N r_{L}}^{D_{1, L}}$ & 0.055 \\
\hline$w_{D_{2, R, \text { Contra }}}^{C T X_{R, I p s i}}$ & 0.02 & $w_{D_{2, R, \text { Contra }}}^{C T X_{R, \text { contra }}}$ & 0.70 & $w_{P V_{R}}^{C T X_{R}}$ & 0.015 & $w_{S N r_{L}}^{D_{2, L}}$ & 0.01 \\
\hline$w_{D_{1, R, I p s i}}^{C T X_{R, I p s i}}$ & 0.60 & $w_{D_{1, R, I} \text { psi }}^{C T X_{R, \text { contra }}}$ & 0.12 & $w_{D_{i, j, k}}^{P V_{j}}$ & 5.88 & $w_{S N r_{R}}^{D_{1, R}}$ & 0.055 \\
\hline$w_{D_{2, R, I}}^{C T X_{R, I p s i}}$ & 0.50 & $w_{D_{2, R, I p s i}}^{C T X_{R, \text { Contra }}}$ & 0.06 & $w_{D_{i, j, k}}^{D_{i, j, k}}$ & 0.60 & $w_{S N r_{R}}^{D_{2, R}}$ & 0.01 \\
\hline$w_{D_{1, L, \text { Contra }}}^{C T X_{L, \text { contra }}}$ & 0.80 & $w_{D_{1, L, \text { contra }}}^{C T X_{L, I p s i}}$ & 0.051 & $\tau_{D_{i j k} \& P V_{j}}$ & 0.05 & $w_{S N r}^{S N r}$ & 0.01 \\
\hline$w_{D_{2, L, \text { Contra }}}^{C T X_{L, \text { contra }}}$ & 0.70 & $w_{D_{2}, L, \text { Contra }}^{C T X_{L, I p s i}}$ & 0.04 & $\tau_{S N r_{j}}$ & 0.10 & $w_{S N r_{R}}^{S N r_{L}}$ & 0.01 \\
\hline$w_{D_{1, L, I p s i}}^{C T X_{L, \text { ontra }}}$ & 0.12 & $w_{D_{1, L, I p s i}}^{C T X_{L, I p s i}}$ & 0.60 & $w_{S N r_{L}}^{S N r_{R}}$ & 0.01 & & \\
\hline$w_{D_{2, L, I p s i}}^{C T X_{L, \text { contra }}}$ & 0.06 & $w_{D_{2, L, I p s i}}^{C T X_{L, I p s i}}$ & 0.456 & $w_{D i^{\prime} j k^{\prime}}^{D i j k}$ & 0.01 & & \\
\hline
\end{tabular}

Extended Data Tab. 2 | Optogenetic perturbation current of left hemispace $(\mu \mathrm{A})$.

\begin{tabular}{c|ccccc}
\hline Type & D1 activation & D2 activation & D1 inactivation & D2 inactivation & PV inactivation \\
\hline$I^{\text {stim }}$ & $10.5 \pm 2.5$ & $10.5 \pm 2.5$ & $-2.5 \pm 1.0$ & $-2.5 \pm 1.0$ & $-2.5 \pm 1.0$ \\
\hline
\end{tabular}

Extended Data Movie 1 | Example video of two-photon imaging during behavioral task

Video clip showing continuous two-photon imaging from the TS of the left hemisphere during the head-fixed mouse performing the behavioral task. The OLED board on the right showing trial information and performance outcome.

\section{Extended Data Movie 2 | Example video of optogenetic stimulation during behavioral}

task

Video clip showing optogenetic activation of dSPNs in the right TS during the mouse 
performing the behavioral task. The OLED board on the right showing trial information and performance outcome. Note that the photostimulation in the third trials shown was accompanied by a wrong choice of the animal. 